\title{
Flavonoids and Related Compounds in Non-Alcoholic Fatty Liver Disease Therapy
}

Sandra Pisonero-Vaquero, ${ }^{1}$ Javier González-Gallego, ${ }^{1,2}$ Sonia Sánchez-Campos, ${ }^{1,2}$ and María Victoria García-Mediavilla ${ }^{1,2^{*}}$

${ }^{1}$ Institute of Biomedicine (IBIOMED), University of León, León, Spain

${ }^{2}$ Centro de Investigación Biomédica en Red de Enfermedades Hepáticas y Digestivas (CIBERehd), Instituto de Salud Carlos III, Madrid, Spain

Running title: Flavonoids and NAFLD

*Address correspondence to this author at the Institute of Biomedicine (IBIOMED), Campus Universitario, 24071, León, Spain; Tel: 34-987-291997; Fax: 34-987-291998; E-mail: mvgarm@unileon.es 
Abstract: Non-alcoholic fatty liver disease (NAFLD), the hepatic manifestation of metabolic syndrome, is one of the most common chronic liver diseases, which may progress to fibrosis, cirrhosis and hepatocellular carcinoma. NAFLD is characterized by the accumulation of lipids in the liver arising from multiple factors: increased fatty acid uptake, increased de novo lipogenesis, reduced fatty acid oxidation and very low density lipoproteins (VLDL) secretion. Most therapeutic approaches for this disease are often directed at reducing body mass index and improving insulin resistance through lifestyle modifications, bariatric surgery and pharmacological treatments. Nevertheless, there is increasing evidence that the use of natural compounds, as polyphenols, exert multiple benefits on the disorders associated with NAFLD. These molecules seem to be able to regulate the expression of genes mainly involved in de novo lipogenesis and fatty acid oxidation, which contributes to their lipid-lowering effect in the liver. Their antioxidant, anti-inflammatory, antifibrogenic and antilipogenic properties seem to confer on them a great potential as strategy for preventing NAFLD progression. In this review, we summarized the effects of these compounds, especially flavonoids, and their mechanisms of action, that have been reported in several studies carried out in in vitro and in vivo models of NAFLD.

Keywords: antioxidant, flavonoids, insulin resistance, lipid accumulation, NAFLD, quercetin. 


\section{INTRODUCTION}

Non-alcoholic fatty liver disease (NAFLD) is one of the most common chronic liver diseases in Western countries and its worldwide prevalence rate ranges from $6 \%$ to $35 \%$, with a median of $20 \%$ [1]. It is considered the hepatic manifestation of the metabolic syndrome [2], ranging from simple steatosis to non-alcoholic steatohepatitis, liver fibrosis, cirrhosis and hepatocellular carcinoma [3]. About $10-20 \%$ of patients who have fatty liver develop inflammation and fibrosis, probably as consequence of a failure of antilipotoxic protection; nevertheless, inflammation may precede steatosis. In both situations, many parallel hits derived from extrahepatic tissues may promote liver inflammation and contribute to its progression to fibrosis and tumor development [4]. The best known risk factors for NAFLD are obesity, hyperglycemia, insulin resistance and hypertriglyceridemia [5].

NAFLD is characterized by the accumulation of lipids in the liver that stems from multiple factors. Thus, the increased fatty acid uptake as a result of the enhancement of the lipolysis from the adipocytes or the increased intake of dietary fat could contribute to this phenomenon. Additionally, increased de novo lipogenesis as well as reduced fatty acid oxidation and very low density lipoproteins (VLDL) secretion contribute to triglyceride accumulation in the liver [6]. However, this accumulation of triglycerides in the form of droplets within the hepatocytes may be protective whereas free fatty acids can serve as substrates for the formation of nontriglyceride lipotoxic metabolites that could cause actually the liver injury [7].

As it is represented in Fig. 1, the main proteins implicated in fatty acid uptake and trafficking are caveolins, fatty acid transport proteins (FATPs), fatty acid translocase (FAT) CD36 and fatty acid binding proteins (FABPs) [6, 8]. A complex network of nuclear receptors regulates enzymes, such as fatty acid synthase (FAS), involved in different steps of hepatic de novo lipogenesis [9]. It is worth mentioning liver $\mathrm{X}$ receptor (LXR), sterol regulatory element binding protein (SREBP)-1c, peroxisome proliferator activated receptor (PPAR) $\gamma$ or carbohydrate responsive element binding protein (ChREBP) $[10,11]$. PPAR $\alpha$ plays a central role in promoting the expression of key enzymes of fatty acid oxidation such as carnitine palmitoyltransferase 1 (CPT1), medium-chain acyl-CoA dehydrogenase (MCAD) or acyl-CoA oxidase (ACO) [12]. Finally, the microsomal triglyceride transfer protein (MTP) regulates triglyceride secretion through VLDL formation [13]. 


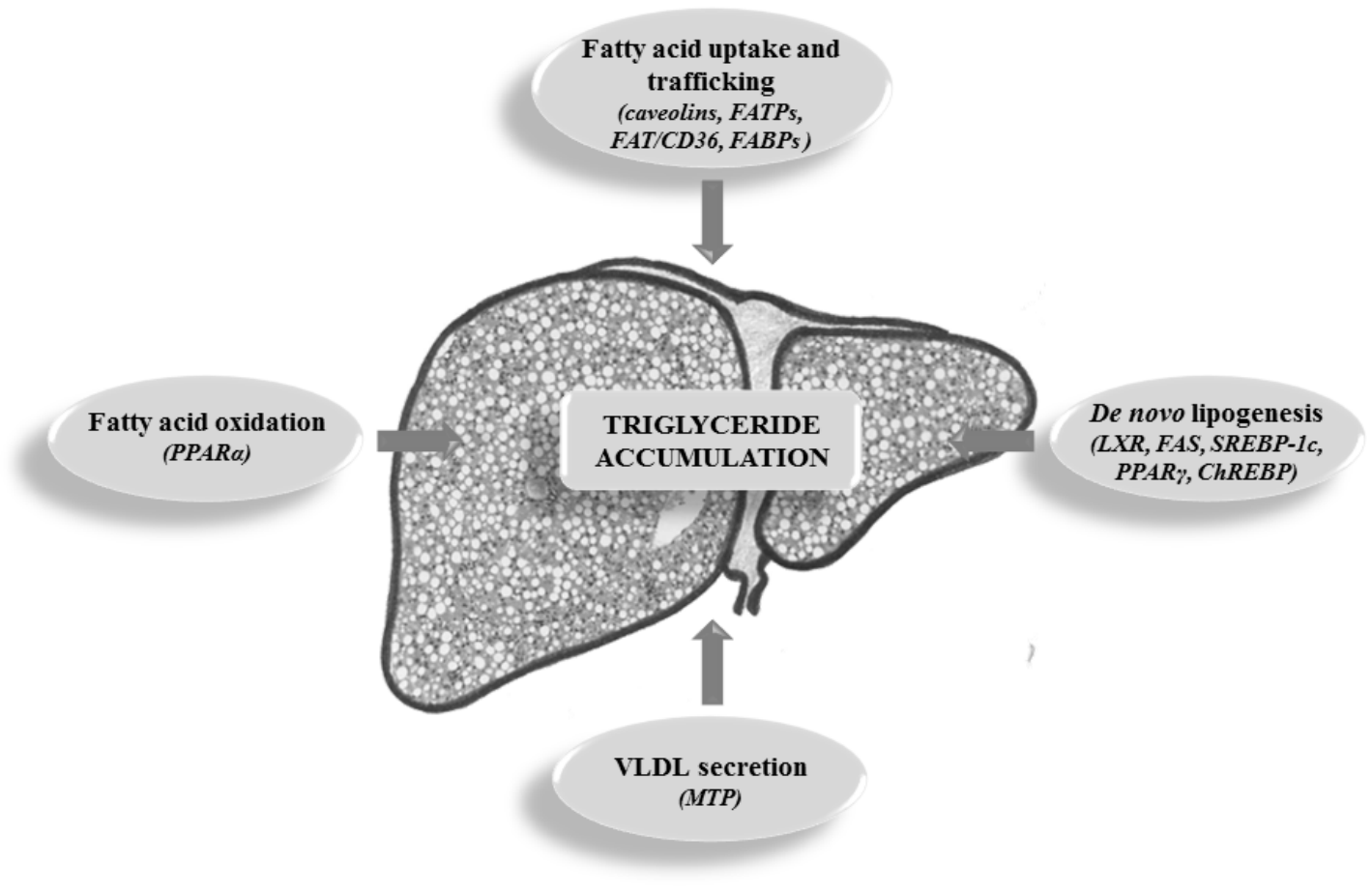

Fig (1). Principal genes that regulate the different processes controlling hepatic triglyceride accumulation.

Most therapies for NAFLD target the major pathways that seem to be essential in the pathogenesis of this disease and are usually directed at reducing body mass index and improving insulin resistance through physical exercise, dietary modification, bariatric surgery and pharmacological treatments [14]. As oxidative stress and inflammation play central roles in NAFLD progression, the use of flavonoids and related compounds with antioxidant and anti-inflammatory properties results in beneficial effects on this and other chronic diseases [15-17]. Moreover, polyphenols can target specific miRNAs that regulate several metabolic pathways including lipid metabolism, which suggests an alternative mechanism by which these compounds could protect from NAFLD [18]. Moreover, development and progression of NAFLD is linked to small intestinal bacterial overgrowth, so supplementation with probiotics seems to improve liver damage, reducing oxidative stress and inflammation by making stronger the intestinal wall, thereby reducing its permeability, bacterial translocation and endotoxemia [19]. In this regard, it has been reported that several flavonoids display prebiotic actions [20], which contribute to their beneficial effects on NAFLD.

These evidences place the use of polyphenolic compounds as an attractive and potential therapeutic strategy for preventing the progression of NAFLD. Their multiple benefits and the different molecular mechanisms underlying their effects are herein reviewed. 


\section{FLAVONOIDS}

Flavonoids are a large class of naturally occurring compounds widely present in fruits, vegetables and beverages derived from plants. The basic structure of these polyphenolic compounds consist of a 15carbon skeleton that can have numerous substituents, determining the subclass of flavonoid and the specific compound within each class (Fig. 2). The effects of the main subclasses of flavonoids (flavones, flavonols, flavanols, isoflavones, flavanones and anthocyanidins) on in vivo models of NAFLD are summarized in Table 1 (flavonols) and Table $\mathbf{2}$ (flavones, flavanones, flavanols, isoflavones and anthocyanidins), while effects on in vitro models of NAFLD are depicted in Table $\mathbf{3}$, being all of them described below.

\subsection{Flavonols}

Quercetin, one of the most studied flavonoids, has a variety of biological functions, including wellknown anti-inflammatory and antioxidant capacities, as well as antiviral activity [17, 21-24]. A comparative study of the role of pioglitazone, quercetin and hydroxy citric acid on steatosis, inflammation, cytochrome P450 (CYP) 2E1 expression and various biochemical parameters in rats fed with high fat diet (HFD), has shown a protective effect of these compounds against non-alcoholic steatohepatitis (NASH), being evident an optimal protection after quercetin treatment compared with pioglitazone or hydroxy citric acid $[25,26]$. Several studies have demonstrated that quercetin is able to improve insulin resistance and hepatic lipid accumulation in different models of NAFLD [16, 27-32]. Recently, it has been reported the involvement of SREBP-1c and FAS upregulation in the increase of the lipid content in an in vitro model of free fatty acid- and insulin-induced NAFLD. After treatment with quercetin at different concentrations, ranging from 0.1 to $100 \mu \mathrm{M}$, the intracellular lipid content was reduced in a dose-dependent manner and the expression of these genes was inhibited, showing that quercetin improved hepatic lipid accumulation by regulating SREBP-1c- and FAS-mediated fatty acid synthesis. Moreover, quercetin induced the phosphorylation of insulin receptor (IR) $\beta$ and insulin receptor substrate 1 (IRS1), which suggests that the flavonoid improves insulin resistance by enhancing insulin signal transduction [27]. This effect on lipid accumulation and insulin resistance has also been observed in similar in vitro models after a treatment with quercetin, showing in addition a reversion of increased lipid peroxidation, DNA fragmentation and inflammatory cytokine secretion, as well as an improvement of antioxidant enzymes levels [28], with a high correlation between inhibitory effects on reactive oxygen species (ROS) generation and intracellular triglyceride levels [33]. In a study about quercetin effect on HFD-induced NAFLD, it has been observed a quercetin-induced regulation of inflammatory cytokines balance that might help to delay the progression of NAFLD through the modulation of liver inflammation and steatosis [34]. These results are in accordance with studies in various nutritional models of NASH that demonstrate an improvement of serum biochemical markers and lipid accumulation in the liver after a treatment with quercetin. The flavonoid is able to reduce biomarkers of liver fibrosis as well as the expression of inflammatory-related genes such as toll-like receptor (TLR)-4, interleukin (IL)-6 or tumor necrosis factor (TNF) $\alpha$. Beneficial effects of quercetin on fibrogenesis and inflammatory state might be related to the reduction of c-Jun terminal kinase (JNK) phosphorylation and the inactivation of nuclear 
factor kappa B (NF-kB)/inducible nitric oxide synthase (iNOS) pathway through the upregulation of silent mating type information regulation 2 homolog 1 (SIRT1) expression, leading to lower inflammatory cytokine transcription [16, 21, 35]. A reduced fibrosis and improvement in liver function has been reported in a model of NAFLD in rats after a treatment with quercetin [29]. Furthermore, in agreement with other studies, authors reported an anti-inflammatory role of quercetin through hepatic NF$\kappa \mathrm{B}$ downregulation, besides an upregulation of nuclear factor (erythroid-derived 2)-related factor-2 (Nrf2) and subsequent heme oxygenase-1 (HO-1) increase, which contribute to cellular stress reduction; moreover, quercetin might attenuate steatosis by increasing fatty acid oxidation through CPT1 upregulation and removing steatotic cells from the liver due to an enhancement of caspase- 3 expressioninduced apoptosis [29]. On the other hand, an inhibitory effect of quercetin on caspase-1 expression, by suppressing the overexpression of hepatic thioredoxin-interacting protein (TXNIP) and subsequent NODlike receptor family pyrin domain-containing 3 (NLRP3) inflammasome activation, could contribute to its lipid-lowering capacity, through downregulation of SREBP-1c and SREBP-2 [32]. Quercetin also reduces plasma and liver free fatty acids, cholesterol and triglyceride levels, by modulating lipid metabolismrelated gene expression, preventing PPAR $\alpha$ reduction, increasing paraoxonase 1 (PON1) and farnesyltransferase/geranylgeranyltransferase type-1 subunit alpha (FNTA) expressions and suppressing expression of aldehyde dehydrogenase (ALDH) 1B1, apolipoprotein (Apo) A4, ATP-binding cassette (ABC) G5, glycerol-3-phosphate acyltransferase 1 mitochondrial (GPAM), acetyl-CoA carboxylase (ACC), farnesyl-diphosphate farnesyltransferase 1 (FDFT1), PPAR $\gamma$, FAS, FAT/CD36, CCAAT/enhancer binding protein (C/EBP) $\alpha$ and LXR $\alpha[30,32]$.

Morin (3,5,7,20,40-pentahydroxyflavone) is a flavonoid isolated from Chinese herbs of the Moraceae family. In high fructose-fed rats morin reduced systemic inflammation, dyslipidemia and insulin and leptin resistance; it also diminished hepatic lipid accumulation through upregulation of PPAR $\alpha$ and CPT1 expression, and downregulation of SREBP-1c and stearoyl-CoA desaturase-1 (SCD1) expression. The elevation of hepatic levels of IL-1 $\beta$, IL- 6 and TNF $\alpha$ in high fructose-fed rats was attenuated by morin through inhibiting hepatic sphingosine kinase 1 (SphK1)/sphingosine 1-phosphate (S1P) signaling pathway and subsequent hepatic NF-kB activation. These effects of morin were afterwards confirmed in a cell model stimulated with fructose [36].

Rutin is a glycoside of quercetin that can be found in onions, apples, tea or red wine. This flavonol slightly reduces ROS generation and triglyceride accumulation in an oleic acid-induced hepatic steatosis in an in vitro model [33]. Rutin is able to effectively attenuate in vivo diet-induced metabolic syndrome, NASH and cardiovascular abnormalities, reducing liver and body weights and plasma triglycerides or free fatty acids and cholesterol levels increase, improving impaired glucose tolerance and antioxidant status, and diminishing steatosis, inflammation and fibrosis in the liver. Rutin could attenuate liver steatosis by upregulating caspase-3-mediated apoptosis of fat-containing hepatocytes, and reduce oxidative stress, as indicated by the increase of heat shock protein (HSP) 70 expression. This flavonol also recovers cardiovascular structure and function, displaying a reduction in systolic blood pressure and an improvement of endothelial function, probably by scavenging free radicals that could in turns increase the nitric oxide bioavailability [37]. Troxerutin, a derivative of rutin, is also able to improve antioxidant 
enzymes levels and hepatic lipid homeostasis, and restore insulin signaling and glycometabolism in HFDinduced NAFLD. The mechanism underlying its beneficial effect could implicate the suppression of oxidative stress-mediated $\mathrm{NAD}^{+}$depletion by increasing nicotinamide phosphoribosyltransferase (NAMPT) expression and decreasing poly (ADP-ribose) polymerase-1 (PARP1), thereby promoting SIRT1-mediated AMP-activated protein kinase (AMPK) activation to inhibit mammalian target of rapamycin (mTOR) signaling; this in turn enhances nuclear lipin1 localization, increasing fatty acid oxidation and triglyceride secretion and suppressing lipogenesis in the liver [38].

Kaempferol, a flavonoid present in several natural sources such as tea, beans, tomatoes, onions, leeks or apples, displays multiple beneficial effects in cardiovascular and nerve systems, and it has also been reported to suppress the lipid accumulation in macrophages through the modulation of the expression of genes implicated in fatty acid uptake such as FAT/CD36 [39]. In vitro studies in HepG2 with inducedlipid accumulation shows that kaempferol, at concentrations ranging from 50 to $150 \mu \mathrm{M}$, presents a high inhibitory effect on cellular triglyceride levels and produces a dose-dependent decrease of intracellular ROS [33].

Isorhamnetin, an O-methylated flavonol aglycone, has been reported to show cardio-protective, antiadipogenic, anti-tumor and anti-inflammatory effects [40-43]. This flavonol is very efficient in reducing triglyceride overaccumulation and ROS generation in vitro after oleic acid-induced hepatic steatosis [33], and it has been suggested that the $\mathrm{Ca}^{2+}$ calmodulin-dependent protein kinase kinase-2 (CaMKK2)mediated AMPK activation is involved in the mechanism underlying the beneficial effect of isorhamnetin in the liver [44].

Tea, berries and other fruits and vegetables contain high concentration of myricetin. This molecule may have a protective effect against diet-induced obesity and insulin resistance partly by improving lipid profile and reducing serum proinflammatory cytokine levels, as well as ameliorating liver steatosis through the increase of fatty acid oxidation by PPAR $\alpha$ upregulation and downregulation of SREBP-1 and SREBP-2 [45, 46], also showing antioxidant and lipid-lowering effects in vitro [33].

The flavonol galangin is the major component of Alpinia officinarum, widely used in traditional Chinese medicine for the treatment of several conditions. The Alpinia officinarum extract is able to efficiently suppress the expressions of C/EBP $\alpha$, FAS, SREBP-1 and PPAR $\gamma$ in the liver and adipose tissue of HFD-fed mice, contributing to its antiobesity effect [47]. Nevertheless, the use of galangin at doses ranging from 1 to $40 \mu \mathrm{M}$ fails to diminish oleic acid-induced triglyceride accumulation in an in vitro model, displaying only a slight reduction of ROS overproduction [33].

\subsection{Flavones}

Chrysin (5,7-di-OH-flavone) has been reported to be involved in various biological activities [48-50], and it seems to activate PPAR $\gamma$ in macrophages [51]. This flavone could ameliorate HFD-induced liver steatosis and inflammation, reducing pro-inflammatory cytokines and inducing anti-inflammatory cytokines and adiponectin levels, via induction of anti-inflammatory M2 phenotype and inhibition of pro- 
inflammatory M1 phenotype of macrophages through PPAR $\gamma$ upregulation [52]. On the contrary, chrysin displays a lacking triglyceride-lowering effect and a pro-oxidant activity in vitro at concentrations of 1-15 $\mu \mathrm{M}[33]$.

Apigenin (4',5,7-trihydroxyflavone) is a flavonoid found in many fruits and vegetables, being parsley and chamomile the most abundant sources. This flavone has been documented to present antiinflammatory [53,54] and anticancer properties [55-57]. In spite of its gluconeogenic and lipogenic reduction-mediated antidiabetic capacity reported in human cells [58], it does not seem to be able to reduce triglyceride overaccumulation in oleic acid-treated HepG2 cells, showing also a remarkable dosedependent induction of ROS levels at concentrations ranging from 10 to $125 \mu \mathrm{M}$ [33].

Luteolin ( $3^{\prime}, 4^{\prime}, 5,7$,-tetrahydroxyflavone) is one of the most common polyphenolic flavonoids present in many edible plants such as carrots, peppers, celery, olive oil, peppermint, thyme, rosemary and oregano [59]. Similarly to apigenin, luteolin presents anti-inflammatory [60] and anticancer [61, 62] properties and a gluconeogenic and lipogenic reduction-mediated antidiabetic capacity in vitro [58], but unlike apigenin, luteolin is able to reduce in vitro ROS production and triglyceride overaccumulation [33, 63], probably through the downregulation of SREBP-1c and FAS gene expressions and the AMPK-mediated upregulation of CPT1 [63].

\subsection{Flavanones}

Naringenin is a citrus-derived flavonoid, that has been reported to attenuate dyslipidemia and improve insulin resistance in addition to steatosis, by increasing hepatic fatty acid oxidation and preventing SREBP-1c-mediated lipogenesis in several in vivo models [64]. Nevertheless, its use in an in vitro model of oleic acid-induced hepatic steatosis fails to decrease triglyceride content and displays a pro-oxidant effect [33].

Another flavonoid found abundantly in citrus fruits is hesperetin. This flavanone has shown antioxidant [65], anti-inflammatory [66], anticancer [67], antiallergic [68] and neuroprotective [69] properties, but little is known about its effect on NAFLD models. A treatment with hesperetin in an in vitro model of hepatic steatosis could not reduce triglyceride increase and slightly suppressed intracellular ROS [33].

The flavanone taxifolin shows a high antioxidant and antilipogenic effects in vitro, reducing the induced triglyceride overaccumulation [33], an effect that could be mediated, at least in part, by its ability to inhibit diacylglycerol acyltransferase (DGAT) and FAS activities [70, 71].

\subsection{Flavanols}

Recently, the effect of (-)-epigallocatechin-3-gallate (EGCG) on steatosis, liver fibrosis and tumorigenesis has been studied using a new model of NASH based on obese and hypertensive rats treated with HFD and carbon tetrachloride. Authors observed a fibrogenesis inhibition effect of EGCG by targeting the activation of the renin-angiotensin system through the reduction of serum levels of 
angiotensin-II (AT-II) and the suppression of angiotensin-converting enzyme (ACE) and AT-II type 1 receptor (AT-1R) expressions. EGCG also reduced inflammation, oxidative stress and lipid peroxidation, preventing NASH-related liver fibrosis and subsequent tumorigenesis as it was demonstrated by the inhibition of the development of hepatic premalignant lesions [72]. Transforming growth factor (TGF)/SMAD, phosphatidylinositol 3-kinase (PI3K)/AKT/forkhead box protein O1 (FOXO1) and NF-kB pathways could be also implicated in the beneficial effect of EGCG on fibrosis, oxidative stress and inflammation [73]. Moreover, EGCG plays a positive role in diminishing triglyceride levels in vitro at concentrations under $25 \mu \mathrm{M}$, but it reflects a negative effect at higher concentrations. As EGCG, the flavanols (+)-catechin and (-)-epicatechin display a triglyceride-lowering effect in the same in vitro model [33].

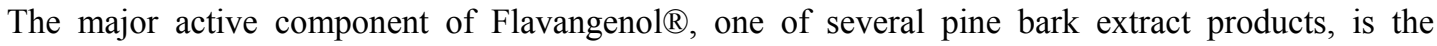
procyanidin B1. This catechin is able to reduce palmitic acid-induce triglyceride accumulation in HepG2 cells by enhancing the $\beta$-oxidation of fatty acids through the gene expression upregulation of key enzymes involved in this process [74].

\subsection{Isoflavones}

In vivo and in vitro studies about beneficial relation of isoflavones and NAFLD and adiposity have been summarized in a review of Kim and Kang [75], putting special emphasis in the effect of these compounds via ChREBP and Wnt signaling.

One of the most prevalent isoflavones in soy is daidzein [76]. It has been reported that daidzein supplementation might alleviate HFD-induced NAFLD in mice by regulating de novo lipogenesis and improving insulin resistance, as well as modulating adipocyte metabolism. The mechanism underlying this effect could implicate LXR $\beta$ and ChREBP downregulation, fatty acid $\beta$-oxidation-related gene expression upregulation and the increase of leptin and adiponectin but the reduction of ghrelin levels [77]. Daidzein is also documented to reduce in a dose-dependent manner both triglyceride and ROS levels in an in vitro model of oleic acid-induced steatosis [33].

The use of puerarin, the major active component isolated from Pueraria lobata, is reported to show beneficial effects in several models of NAFLD. This isoflavone is able to diminish steatosis, inflammation and ballooning by reducing triglyceride and cholesterol levels and TNF $\alpha$ expression in the liver, and improving leptin signal transduction through janus kinase 2 (JAK2)/signal transducer and activator of transcription 3 (STAT3) pathways [78-80].

Another of most abundant isoflavones in soy is genistein [76]. Its use in in vitro [33] and in vivo [81, 82] models reveals multiple benefits in NAFLD. Genistein reduces body and liver weights increase and visceral fat pads, improves insulin resistance, serum lipid profile, antioxidant status and NAFLD activity score by diminishing cholesterol, triglycerides and free fatty acids in the liver in addition to TNF $\alpha$ and IL6 gene expressions. Moreover, it induces an increase in the expression of adiponectin, which functions as an anti-fibrogenic and anti-inflammatory cytokine. The enhancement of fatty acid $\beta$-oxidation and the 
reduction of lipogenesis in adipose tissue, mediated by the upregulation of AMPK, PPAR $\alpha$ and very longchain acyl-CoA dehydrogenase (VLCAD) and the repression of ACC2, retinoid X receptor (RXR) $\alpha$, LXR $\alpha$, SREBP-1c, C/EBP $\beta$ and PPAR $\gamma$ could also contribute to the beneficial effects of genistein, leading to a diminution of visceral fat mass [82].

\subsection{Anthocyanidins}

Cyanidin is an anthocyanidin commonly found in fruits and vegetables. The antioxidant enzyme activation through extracellular signal-regulated kinase (ERK) and JNK pathways and Nrf2 upregulation in addition to ameliorated cytotoxicity and the improvement of the perturbation of genes involved in lipid metabolism, could contribute to its beneficial effects on NASH [83].

A glycoside of cyanidin, cyanidin-3-O- $\beta$-glucoside, is able to improve obesity and triglyceride metabolism in KKAy mice, reducing body and liver weights and fat pads, and hepatic and serum triglyceride content. These actions may be mediated by an increase in lipoprotein lipase (LPL) activity in plasma and skeletal muscle and inhibition of LPL in adipose tissue through the activation of AMPK [84]. Furthermore, this anthocyanin decreases hepatic glycerol-3-phosphate acyltransferase 1 (GPAT1) activity and its translocation to the outer mitochondrial membrane, diminishing triglyceride synthesis and thereby attenuating hepatic steatosis [85]. In vitro studies show the hepatoprotective effect of cyanidin-3-O- $\beta$ glucoside against hyperglycemia-accelerated steatohepatitis in NAFLD, process mediated, at least in part, by the improvement of antioxidant status and the inhibition of the mitochondria-mediated apoptotic pathway through AKT activation and JNK inactivation [86]. This anthocyanin has been also used combined with others such as cyanidin-3-rutinoside and pelargonidin-3-glucoside, displaying a protective effect against diet-induced body weight gain [87].

More recently, a further review about the effect of anthocyanins on hepatic lipid accumulation and lipotoxicity associated with NAFLD in both in vitro and in vivo models, has proposed the possible mechanism underlying the beneficial effects of anthocyanins, based on available studies. This includes the AMPK activation-mediated lipogenesis inhibition and lipolysis promotion by reducing SREBP-1c and inducing PPAR $\alpha$ activity respectively, in addition to the reduction of oxidative stress by the induction of antioxidant enzymes [88]. 

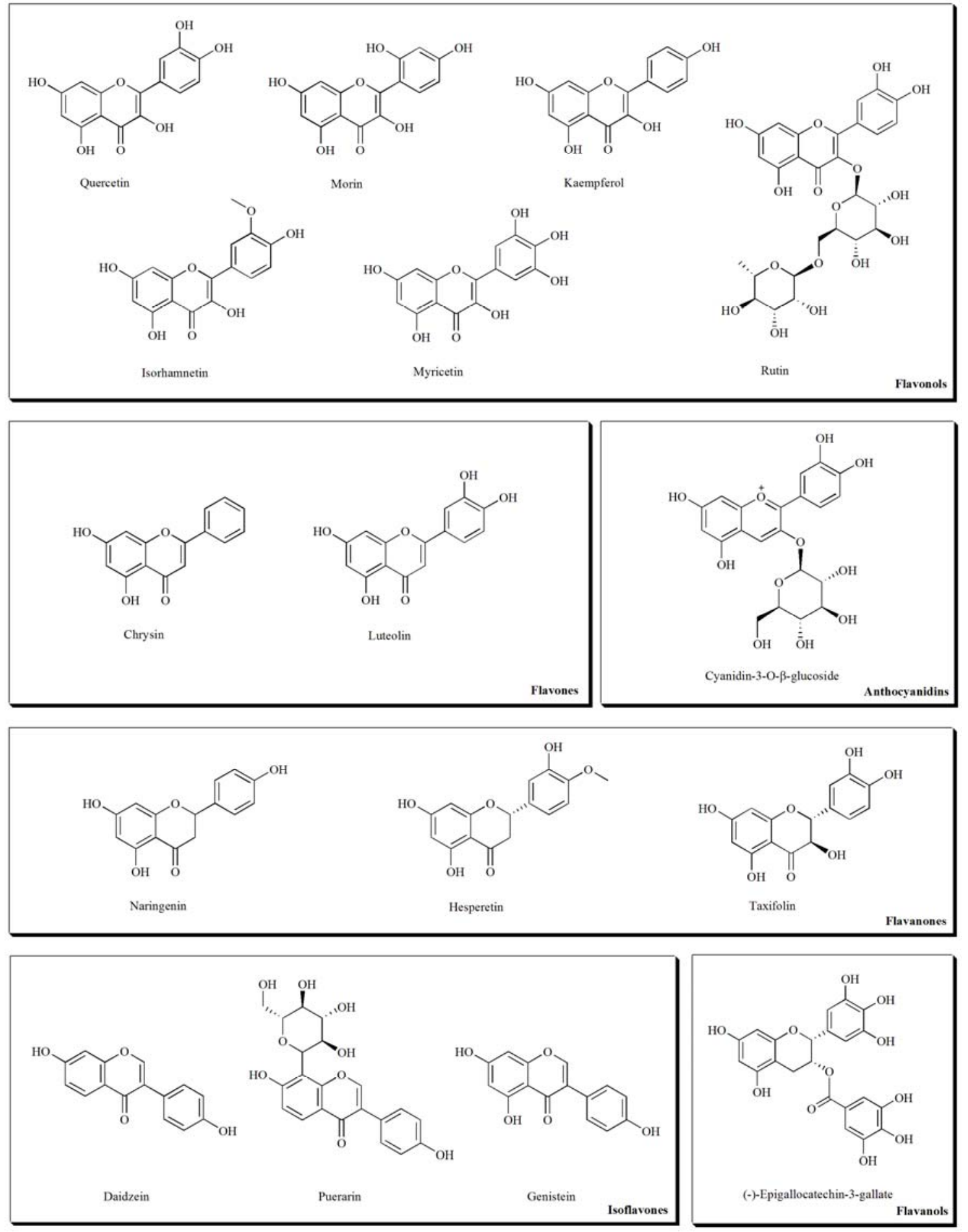

Fig (2). Representative compounds of the major classes of flavonoids with known effects on NAFLD. 
Table 1. Effects of flavonols on in vivo models of NAFLD.

\begin{tabular}{|c|c|c|c|c|c|}
\hline Flavonoid treatment & Model & Physiological variables & Blood parameters & Histopathology and gene expression & Ref. \\
\hline $\begin{array}{l}\text { Quercetin } 20 \mathrm{mg} / \mathrm{kg} \text { b.w./day } \\
\text { (last } 4 \text { weeks) }\end{array}$ & $\begin{array}{l}\text { Wistar rats. HFD feeding } \\
\text { (8 weeks) }\end{array}$ & & $\begin{array}{l}\uparrow \mathrm{AST}, \mathrm{ALT}, \gamma \mathrm{GT}(\mathrm{Q} \downarrow) \\
\uparrow \mathrm{LDL}(\mathrm{Q} \downarrow) \\
\uparrow \text { Glucose }(\mathrm{Q} \downarrow) \\
\uparrow \text { Albumin, creatinine, urea, uric acid, } \\
\text { bilirubin }(\mathrm{Q} \downarrow)\end{array}$ & $\begin{array}{l}\uparrow \text { Steatosis }(\mathrm{Q} \downarrow) \\
\uparrow \text { Inflammation }(\mathrm{Q} \downarrow) \\
\uparrow \text { CYP2E1 }(\mathrm{Q} \downarrow)\end{array}$ & {$[25,26]$} \\
\hline $\begin{array}{l}\text { Quercetin } 75 \text { and } 300 \mathrm{mg} / \mathrm{Kg} \\
\text { b.w./day (last } 8 \text { weeks) }\end{array}$ & $\begin{array}{l}\text { Sprague-Dawley rats. HFD } \\
\text { feeding ( } 12 \text { weeks) }\end{array}$ & $\uparrow$ Liver index $(\mathrm{Q} \downarrow)$ & $\begin{array}{l}\uparrow I \mathrm{IL}-18(\mathrm{Q} \downarrow) \\
\downarrow I \mathrm{~L}-10(\mathrm{Q} \uparrow) \\
\uparrow \text { Glucose }(\mathrm{Q} \downarrow)\end{array}$ & $\begin{array}{l}\uparrow \text { Steatosis }(\mathrm{Q} \downarrow) \\
\uparrow \text { Inflammation }(\mathrm{Q} \downarrow)\end{array}$ & [34] \\
\hline $\begin{array}{l}\text { Quercetin } 15,30 \text { and } 60 \\
\mathrm{mg} / \mathrm{Kg} \text { b.w./day (last } 2 \text { weeks) }\end{array}$ & $\begin{array}{l}\text { Gerbils. HFD feeding ( } 4 \\
\text { weeks) }\end{array}$ & & $\begin{array}{l}\uparrow \mathrm{AST}, \mathrm{ALT}(\mathrm{Q} \downarrow) \\
\uparrow \mathrm{TG}, \text { cholesterol }(\mathrm{Q} \downarrow) \\
\uparrow \mathrm{LDL}(\mathrm{Q} \downarrow) \\
\uparrow \text { Glucose }(\mathrm{Q} \downarrow) \\
\text {--Albumin, bilirubin, ammonia (Q--) } \\
\uparrow T N F \alpha, \text { IL-6 }(\mathrm{Q} \downarrow)\end{array}$ & $\begin{array}{l}\uparrow \text { Fibrosis }(\mathrm{Q} \downarrow) \\
\uparrow \text { Steatosis }(\mathrm{Q} \downarrow) \\
\uparrow \text { Inflammation }(\mathrm{Q} \downarrow) \\
\downarrow \text { SIRT1 }(\mathrm{Q} \uparrow) \\
\uparrow \mathrm{NF}-\mathrm{kB} \text { p65, iNOS }(\mathrm{Q} \downarrow)\end{array}$ & [16] \\
\hline $\begin{array}{l}\text { Quercetin } 50 \mathrm{mg} / \mathrm{Kg} \text { b.w./day } \\
\text { (4 weeks) }\end{array}$ & $\begin{array}{l}\text { C57BL/6J mice. MCD- } \\
\text { feeding ( } 4 \text { weeks) }\end{array}$ & $\begin{array}{l}\downarrow \text { Body weight (Q--) } \\
\downarrow \text { Liver weight (Q--) }\end{array}$ & $\uparrow \mathrm{AST}, \mathrm{ALT}(\mathrm{Q} \downarrow)$ & $\begin{array}{l}\uparrow N A S(Q \downarrow) \\
\uparrow T B A R S(Q \downarrow) \\
\uparrow \alpha-S M A(Q \downarrow) \\
\uparrow T L R-4, \text { HMGB1, IL-6, TNF } \alpha, \text { COX-2 (Q } \downarrow) \\
\uparrow C O L 1 A 1, \text { COL3A1, PLOD3, TGF } 11, \text { SMAD3, SMAD7, TIMP1, } \\
\text { MMP9, CTGF, AREG, PDGFB (Q } \downarrow) \\
\uparrow N F-\kappa B \text { p65, pJNK (Q } \downarrow)\end{array}$ & [21] \\
\hline $\begin{array}{l}\text { Quercetin } 800 \mathrm{mg} / \mathrm{Kg} \text { food } \\
\text { (last } 8 \text { weeks) }\end{array}$ & $\begin{array}{l}\text { Wistar rats. High } \\
\text { carbohydrate HFD-feeding } \\
\text { (16 weeks) }\end{array}$ & $\begin{array}{l}\uparrow \text { Body weight }(\mathrm{Q}--) \\
\uparrow \text { Liver weight }(\mathrm{Q} \downarrow) \\
\uparrow \text { BMI (Q--) } \\
\uparrow \text { Fat pads }(\mathrm{Q} \downarrow)\end{array}$ & $\begin{array}{l}\uparrow A L T, \text { ALP, LDH }(\mathrm{Q} \downarrow) \\
\uparrow A S T(\mathrm{Q}--) \\
\uparrow T G(\mathrm{Q} \uparrow) \\
\uparrow F F A, \text { cholesterol (Q--) } \\
\uparrow \mathrm{Glucose}(\mathrm{Q} \downarrow) \\
\downarrow \text { Urea }(\mathrm{Q} \uparrow) \\
\uparrow \text { Uric acid }(\mathrm{Q} \downarrow) \\
\text {--Bilirubin }(\mathrm{Q} \downarrow) \\
\end{array}$ & 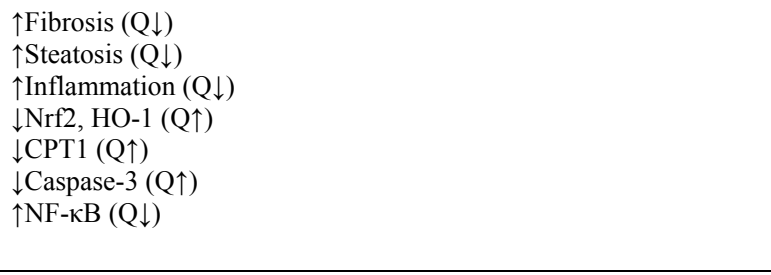 & [29] \\
\hline $\begin{array}{l}\text { Quercetin } 250 \mathrm{mg} / \mathrm{Kg} \text { food ( } 9 \\
\text { weeks) }\end{array}$ & $\begin{array}{l}\text { C57BL/6 J mice. HFD- } \\
\text { feeding ( } 9 \text { weeks) }\end{array}$ & $\begin{array}{l}\uparrow \text { Body weight }(\mathrm{Q} \downarrow) \\
\uparrow \text { Liver weight }(\mathrm{Q} \downarrow)\end{array}$ & $\begin{array}{l}\uparrow T G, \text { cholesterol }(\mathrm{Q} \downarrow) \\
\uparrow T B A R S(Q \downarrow)\end{array}$ & $\begin{array}{l}\uparrow \text { Steatosis }(\mathrm{Q} \downarrow) \\
\uparrow T G(\mathrm{~T} \downarrow) \\
\downarrow \text { CYP2C50, PON1, FNTA, PPAR } \alpha, \text { PPAR } \delta(\mathrm{Q} \uparrow) \\
\uparrow \text { ALDH1B1, ApoA4, ABCG5, GPAM, ACC, FDFT1, VCAM1, } \\
\text { PPAR } \gamma, \text { FAT/CD36, FAS, C/EBP } \alpha(\mathrm{Q} \downarrow)\end{array}$ & [30] \\
\hline
\end{tabular}




\begin{tabular}{|c|c|c|c|c|c|}
\hline $\begin{array}{l}\text { Quercetin } 75 \text { and } 300 \mathrm{mg} / \mathrm{Kg} \\
\text { b.w./day (last } 8 \text { weeks) }\end{array}$ & $\begin{array}{l}\text { Sprague-Dawley rats. } \\
\text { HFD-feeding (12 weeks) }\end{array}$ & & $\begin{array}{l}\text { Resistin }(\mathrm{Q} \downarrow) \\
\text { IL-18 }(\mathrm{Q} \downarrow) \\
\text { Glucose, insulin }(\mathrm{Q} \downarrow) \\
\text { HOMA-IR }(\mathrm{Q} \downarrow) \\
\text { TG }(\mathrm{Q} \downarrow) \\
\text { MDA }(\mathrm{Q} \downarrow)\end{array}$ & & [31] \\
\hline $\begin{array}{l}\text { Quercetin } 25,50 \text { and } 100 \\
\mathrm{mg} / \mathrm{Kg} \text { b.w./day (7 weeks) }\end{array}$ & $\begin{array}{l}\text { Sprague-Dawley rats. } \\
\text { Streptozotocin-induced } \\
\text { diabetes }\end{array}$ & $\begin{array}{l}\downarrow \text { Body weight }(\mathrm{Q} \uparrow) \\
\uparrow \text { Liver weight }(\mathrm{Q} \downarrow)\end{array}$ & $\begin{array}{l}\uparrow A S T, \text { ALT, ALP }(\mathrm{Q} \downarrow) \\
\uparrow \mathrm{TG}, \mathrm{FFA} \text {, cholesterol }(\mathrm{Q} \downarrow) \\
\uparrow \mathrm{Glucose}(\mathrm{Q} \downarrow) \\
\uparrow \mathrm{IL}-18, \mathrm{IL}-1 \beta(\mathrm{Q} \downarrow)\end{array}$ & $\begin{array}{l}\uparrow N A S(Q \downarrow) \\
\uparrow T G, F F A, \text { cholesterol }(\mathrm{Q} \downarrow) \\
\uparrow I L-18, \text { IL-1 } \beta(Q \downarrow) \\
\uparrow R O S(Q \downarrow) \\
\uparrow X O, X D H, X O / X D H(Q \downarrow) \\
\uparrow T X N I P, \text { NLRP3, ASC, caspase-1 }(\mathrm{Q} \downarrow) \\
\uparrow \operatorname{SREBP-1c,~SREBP-2,~FAS,~LXR~} \alpha(Q \downarrow) \\
\downarrow P P A R \alpha(Q \uparrow)\end{array}$ & {$[32]$} \\
\hline Quercetin (2 weeks) & $\begin{array}{l}\text { Swiss mice. HFD-feeding } \\
\text { ( } 2 \text { weeks) }\end{array}$ & & & $\begin{array}{l}\uparrow \mathrm{ROS}(\mathrm{Q} \downarrow) \\
\downarrow \text { Antioxidant profile }(\mathrm{Q} \uparrow) \\
\uparrow \text { Lipid peroxidation }(\mathrm{Q} \downarrow) \\
\uparrow \text { Inflammation }(\mathrm{Q} \downarrow) \\
\uparrow \mathrm{NF}-\mathrm{kB}(\mathrm{Q} \downarrow) \\
\end{array}$ & {$[35]$} \\
\hline $\begin{array}{l}\text { Morin } 30 \text { and } 60 \mathrm{mg} / \mathrm{Kg} \\
\text { b.w./day (last } 4 \text { weeks) }\end{array}$ & $\begin{array}{l}\text { Sprague-Dawley rats. High } \\
\text { fructose diet ( } 10 \% \text { in } \\
\text { drinking water) ( } 8 \text { weeks) }\end{array}$ & --Body weight (M--) & $\begin{array}{l}\uparrow T G \text {, cholesterol (M } \downarrow \text { ) } \\
\uparrow V L D L(M \downarrow) \\
\uparrow \text { Glucose, insulin }(\mathrm{M} \downarrow) \\
\uparrow \text { Leptin (M } \downarrow) \\
\uparrow T N F \alpha, \text { IL-6, IL-1 } \beta(\mathrm{M} \downarrow)\end{array}$ & 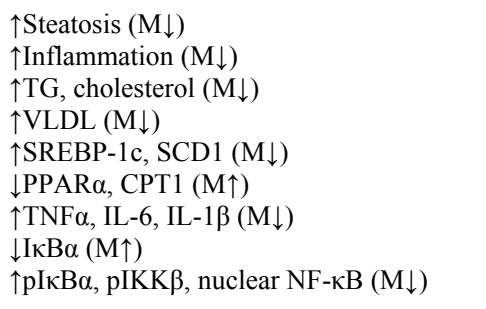 & [36] \\
\hline $\begin{array}{l}\text { Rutin } 1.6 \mathrm{~g} / \mathrm{Kg} \text { food (last } 8 \\
\text { weeks) }\end{array}$ & $\begin{array}{l}\text { Wistar rats. High } \\
\text { carbohydrate HFD-feeding } \\
\text { (16 weeks) }\end{array}$ & $\begin{array}{l}\uparrow \text { Body weight }(\mathrm{R} \downarrow) \\
\uparrow \text { Liver weight }(\mathrm{R} \downarrow) \\
\uparrow \mathrm{BMI}(\mathrm{R} \downarrow) \\
\uparrow \text { Fat pads }(\mathrm{R} \downarrow)\end{array}$ & 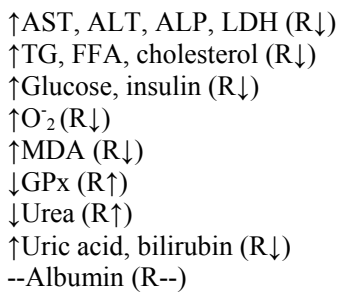 & $\begin{array}{l}\uparrow \text { Fibrosis }(\mathrm{R} \downarrow) \\
\uparrow \text { Steatosis }(\mathrm{R} \downarrow) \\
\uparrow \text { Inflammation }(\mathrm{R} \downarrow) \\
\downarrow \text { Caspase-3, HSP70, ERK1/2(R } \uparrow)\end{array}$ & [37] \\
\hline
\end{tabular}




\begin{tabular}{|c|c|c|c|c|c|}
\hline $\begin{array}{l}\text { Troxerutin } 150 \mathrm{mg} / \mathrm{Kg} \\
\text { b.w./day ( } 20 \text { weeks) }\end{array}$ & $\begin{array}{l}\text { ICR mice. HFD-feeding } \\
(20 \text { weeks })\end{array}$ & $\begin{array}{l}\uparrow \text { Body weight }(\mathrm{Tx} \downarrow) \\
\uparrow \text { Liver index }(\mathrm{Tx} \downarrow) \\
\uparrow \text { Fat pads }(\operatorname{Tx} \downarrow)\end{array}$ & $\begin{array}{l}\uparrow \text { TG, FFA, cholesterol (Tx } \downarrow \text { ) } \\
\uparrow \text { Glucose, insulin }(T x \downarrow) \\
\uparrow A L T \text { (Tx } \downarrow \text { ) }\end{array}$ & $\begin{array}{l}\uparrow N A S(T x \downarrow) \\
\uparrow T G, \text { FFA, cholesterol }(T x \downarrow) \\
\uparrow R O S(T x \downarrow) \\
\uparrow 4-H N E(T x \downarrow) \\
\downarrow \text { GSH, GPx, SOD1 (Tx } \uparrow) \\
\left.\downarrow N A D^{+}, \text {NAMPT, SIRT1, pAMPK, p-raptor, nuclear lipin1 (Tx } \uparrow\right) \\
\uparrow P A R P 1, p-m T O R, \text { cytoplasmic lipin } 1 \text { (Tx } \downarrow \text { ) } \\
\downarrow \text { PPAR } \alpha, \text { PGC1 } \alpha, \text { CPT1A, pACC, ApoB100 (Tx } \uparrow) \\
\uparrow S R E B P-1 c, \text { FAS }(T x \downarrow)\end{array}$ & [38]. \\
\hline $\begin{array}{l}\text { Myricetin } 0.6 \text { and } 1 \mathrm{~g} / \mathrm{Kg} \text { food } \\
\text { (12 weeks) }\end{array}$ & $\begin{array}{l}\text { C57BL/6J mice. High } \\
\text { sucrose HFD-feeding (12 } \\
\text { weeks) }\end{array}$ & $\begin{array}{l}\uparrow \text { Body weight (My } \downarrow \text { ) } \\
\uparrow \text { Fat pads (My } \downarrow \text { ) }\end{array}$ & 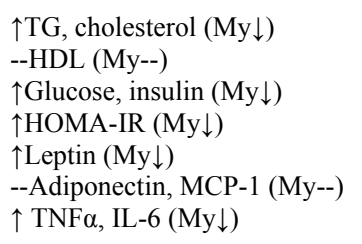 & & [45] \\
\hline $\begin{array}{l}\text { Myricetin } 75,150 \text { and } 300 \\
\mathrm{mg} / \mathrm{Kg} \text { b.w./day (last } 8 \text { weeks) }\end{array}$ & $\begin{array}{l}\text { Wistar rats. HFD-feeding } \\
\text { (10 weeks) }\end{array}$ & $\begin{array}{l}\uparrow \text { Body weight }(\mathrm{My} \downarrow) \\
\uparrow \text { Fat pads (My } \downarrow)\end{array}$ & $\begin{array}{l}\uparrow T G, \text { FFA, cholesterol (My } \downarrow \text { ) } \\
\uparrow \mathrm{LDL}(\mathrm{My} \downarrow) \\
\downarrow \text { HDL (My } \uparrow\end{array}$ & $\begin{array}{l}\uparrow \text { Steatosis }(\mathrm{My} \downarrow) \\
\uparrow T \mathrm{TG}, \text { cholesterol (My } \downarrow \text { ) } \\
\downarrow \text { PPAR } \alpha, \text { ACO, CYP4A (My } \uparrow) \\
\uparrow \text { SREBP-1, SREBP-2 (My } \downarrow \text { ) }\end{array}$ & [46] \\
\hline
\end{tabular}

4-HNE, 4-hydroxy-2'-nonenal; ABC, ATP-binding; ACC, acetyl-CoA carboxylase; ACO, acyl-CoA oxidase; ALDH, aldehyde dehydrogenase; ALP, alkaline phosphatase; ALT, alanine aminotransferase; AMPK, AMP-activated protein kinase; Apo, apolipoprotein; AREG, amphiregulin; ASC, apoptosis-associated speck-like protein containing a caspase recruitment domain; AST, aspartate aminotransferase; BMI, body mass index; C/EBPa, CCAAT/enhancer binding protein alpha; COL, collagen; COX-2, cyclooxygenase-2; CPT1, carnitine palmitoyltransferase 1; CTGF, connective tissue growth factor; CYP, cytochrome P450; ERK, extracellular signal-regulated kinase; FAS, fatty acid synthase; FAT/CD36, fatty acid translocase CD36; FDFT1, farnesyl-diphosphate farnesyltransferase 1; FFA, free fatty acid; FNTA, farnesyltransferase/geranylgeranyltransferase type-1 subunit alpha; GPAM, glycerol-3-phosphate acyltransferase 1 mitochondrial; GPx, glutathione peroxidase; GSH, glutathione; HDL, high density lipoprotein; HFD, high fat diet; HMGB1, high-mobility group box 1 protein; HO-1, heme oxygenase-1; HOMA-IR, homeostasis model assessment for insulin resistance; HSP, heat shock protein; IKKß, inhibitor $\kappa B$ kinase $\beta$; IL, interleukin; iNOS, inducible nitric oxide synthase; IKBa, inhibitor of NF-кB alpha; JNK, c-Jun terminal kinase; LDH, lactate dehydrogenase; LDL, low density lipoprotein; LXRa, liver X receptor alpha; M, morin; MCD, methionine and choline deficient; MDA, malondialdehyde; MMP, matrix metalloproteinase; mTOR, mammalian target of rapamycin; My, myricetin; NAMPT, nicotinamide phosphoribosyltransferase; NAS, NAFLD activity score; NF-KB, nuclear factor kappa B; NLRP3, NOD-like receptor family, pyrin domain-containing 3; Nrf2, nuclear factor (erythroid-derived 2)-related factor-2; PARP, poly (ADP-ribose) polymerase; PDGFB, platelet-derived growth factor subunit B; PGC1a, PPAR $\gamma$ coactivador-1 alpha; PLOD3, procollagen-lysine, 2-oxoglutarate 5-dioxygenase 3; PON1, paraoxonase 1; PPARa, peroxisome proliferator-activated receptor alpha; PPAR $\boldsymbol{\delta}$, peroxisome proliferator-activated receptor delta; PPAR $\boldsymbol{\gamma}$, peroxisome proliferator-activated receptor gamma; $\mathbf{Q}$, quercetin; R, rutin; Raptor, regulatory associated protein of mTOR; ROS, reactive oxygen species; SCD1, stearoyl-CoA desaturase-1; SIRT1, silent mating type information regulation 2 homolog1; SOD, superoxide dismutase; SREBP, sterol regulatory element-binding protein; TBARS, thiobarbituric acid-reactive substance; TG, triglyceride; TGF, transforming growth factor; TIMP, tissue inhibitor of metalloproteinases; TLR, toll-like receptor; TNFa, tumor necrosis factor alpha; Tx, troxerutin; TXNIP, thioredoxin-interacting protein; VCAM1, vascular cell adhesion protein 1; VLDL, very low density lipoprotein; XDH, xanthine dehydrogenase; XO, xanthine oxidase; $\boldsymbol{\alpha}$-SMA, alpha-smooth

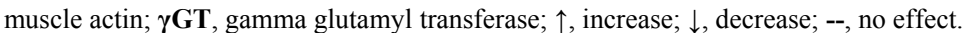


Table 2. Effects of flavones, flavanones, flavanols, isoflavones and anthocyanidins on in vivo models of NAFLD.

\begin{tabular}{|c|c|c|c|c|c|c|}
\hline Group & Flavonoid treatment & Model & Physiological variables & Blood parameters & Histopathology and gene expression & Ref. \\
\hline 冚 & $\begin{array}{l}\text { Chrysin } 25 \text { and } 30 \mathrm{mg} / \mathrm{Kg} \\
\text { b.w./day (last } 2 \text { weeks) }\end{array}$ & $\begin{array}{l}\text { C57BL/6J mice. HFD- } \\
\text { feeding (17 weeks) }\end{array}$ & $\uparrow$ Body weight (C--) & $\begin{array}{l}\uparrow \mathrm{AST}, \mathrm{ALT}(\mathrm{C} \downarrow) \\
\uparrow \mathrm{IL}-1 \beta, \mathrm{TNF} \alpha(\mathrm{C} \downarrow) \\
\downarrow \mathrm{IL}-10(\mathrm{C} \uparrow) \\
\downarrow \text { Adiponectin }(\mathrm{C} \uparrow)\end{array}$ & $\begin{array}{l}\uparrow \text { Steatosis }(\mathrm{C} \downarrow) \\
\uparrow \text { Inflammation }(\mathrm{C} \downarrow) \\
\uparrow \operatorname{PPAR} \gamma(\mathrm{C} \uparrow)\end{array}$ & {$[52]$} \\
\hline \multirow{2}{*}{ 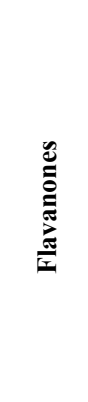 } & $\begin{array}{l}\text { Naringenin } 10 \text { and } 30 \mathrm{~g} / \mathrm{Kg} \\
\text { food (4 weeks) }\end{array}$ & $\begin{array}{l}\text { Ldlr }^{-/} \text {mice. HFD-feeding } \\
\text { (4 weeks) }\end{array}$ & 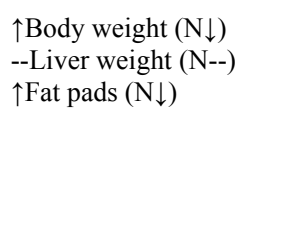 & 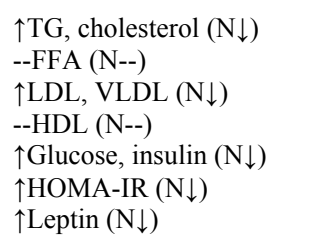 & $\begin{array}{l}\uparrow \text { Steatosis }(\mathrm{N} \downarrow) \\
\uparrow \text { Cholesterol, TG }(\mathrm{N} \downarrow) \\
\uparrow \text { SREBP-1c }(\mathrm{N} \downarrow) \\
\downarrow \text { PGC1 } \alpha(\mathrm{N} \uparrow) \\
\text {--PPAR } \alpha(\mathrm{N}--) \\
- \text {--ACO }(\mathrm{N} \uparrow)\end{array}$ & [64] \\
\hline & $\begin{array}{l}\text { Naringenin } 10 \text { and } 30 \mathrm{~g} / \mathrm{Kg} \\
\text { food ( } 30 \text { weeks) }\end{array}$ & $\begin{array}{l}\text { C57BL/6J mice. HFD- } \\
\text { feeding ( } 30 \text { weeks) }\end{array}$ & $\begin{array}{l}\uparrow \text { Body weight }(\mathrm{N} \downarrow) \\
\uparrow \text { Liver weight }(\mathrm{N} \downarrow) \\
\uparrow \text { Fat pads }(\mathrm{N} \downarrow)\end{array}$ & $\begin{array}{l}\uparrow T G, \text { cholesterol }(\mathrm{N} \downarrow) \\
\uparrow L D L, \text { VLDL }(\mathrm{N} \downarrow) \\
\text {--HDL (N--) } \\
\uparrow \text { Glucose, insulin }(\mathrm{N} \downarrow)\end{array}$ & $\uparrow \mathrm{TG}$, cholesterol $(\mathrm{N} \downarrow)$ & {$[64]$} \\
\hline \multirow{2}{*}{ 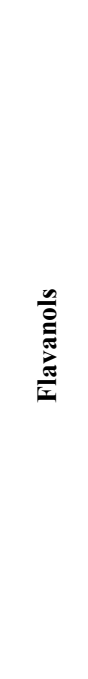 } & $\begin{array}{l}\text { EGCG } 0.1 \% \text { in drinking water } \\
\text { ( } 8 \text { weeks) }\end{array}$ & $\begin{array}{l}\text { SHRSP-ZF rats. HFD- } \\
\text { feeding ( } 8 \text { weeks) and } \mathrm{CCl}_{4} \\
\text { (twice a week, } 8 \text { weeks) }\end{array}$ & $\begin{array}{l}\uparrow \text { Body weight }(\mathrm{EGCG} \uparrow) \\
\uparrow \text { Liver weight }(\mathrm{EGCG} \uparrow)\end{array}$ & $\begin{array}{l}\uparrow \text { AST, ALT (EGCG--) } \\
\uparrow T G \text {, cholesterol (EGCG--) } \\
\uparrow F F A \text { (EGCG } \downarrow \text { ) } \\
\uparrow \text { Glucose, insulin (EGCG--) } \\
\uparrow \text { Leptin (EGCG--) } \\
\downarrow \text { Adiponectin (EGCG--) } \\
\uparrow T N F \alpha, \text { IL-6 (EGCG--) } \\
\uparrow \text { Hydroxyproline (EGCG } \downarrow \text { ) }\end{array}$ & $\begin{array}{l}\uparrow N A S \text { (EGCG } \downarrow \text { ) } \\
\uparrow \text { Fibrosis (EGCG } \downarrow \text { ) } \\
\uparrow \text { Hydroxyproline (EGCG } \downarrow \text { ) } \\
\uparrow \text { MDA, 4-HNE (EGCG } \downarrow \text { ) } \\
\uparrow 8 \text {-OHdG (DNA damage) (EGCG } \downarrow \text { ) } \\
\uparrow \alpha-\text { SMA (EGCG } \downarrow \text { ) } \\
\uparrow \text { MMP2, MMP9, TIMP1, TIMP2, procollagen-1, TGF } \beta 1 \text {, } \\
\text { PAI-1 (EGCG } \downarrow) \\
\uparrow C Y P 2 E 1, \text { pJNK (EGCG } \downarrow \text { ) } \\
\uparrow \text { TNF } \alpha \text {, IL-6, IL-1 } \beta, \text { MCP-1 (EGCG } \downarrow \text { ) } \\
\downarrow \text { CAT, GPx (EGCG } \uparrow \text { ) }\end{array}$ & {$[72]$} \\
\hline & $\begin{array}{l}\text { EGCG } 50 \mathrm{mg} / \mathrm{Kg} \text { b.w./day ( } 3 \\
\text { times per week, } 8 \text { weeks) }\end{array}$ & $\begin{array}{l}\text { Sprague-Dawley rats. } \\
\text { HFD-feeding ( } 8 \text { weeks) }\end{array}$ & --Body weight (EGCG $\downarrow)$ & $\uparrow \mathrm{ALT} / \mathrm{AST}(\mathrm{EGCG} \downarrow)$ & 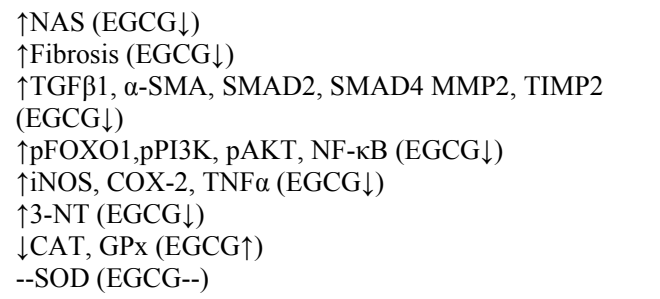 & {$[73]$} \\
\hline
\end{tabular}




\begin{tabular}{|c|c|c|c|c|c|c|}
\hline \multirow{5}{*}{ 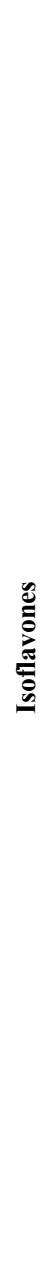 } & $\begin{array}{l}\text { Daidzein } 0.1-2 \mathrm{~g} / \mathrm{Kg} \text { food (12 } \\
\text { weeks) }\end{array}$ & $\begin{array}{l}\text { C57BL/6J mice. HFD- } \\
\text { feeding (12 weeks) }\end{array}$ & & 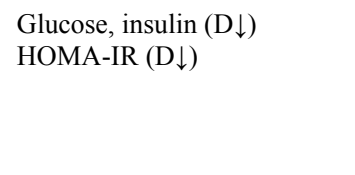 & $\begin{array}{l}\uparrow \text { Steatosis (D } \downarrow) \\
\text { LXR } \beta \text {, ChREBP (D } \downarrow \text { ) } \\
\text { TNF } \alpha(D \downarrow \text { ) } \\
\text { Leptin, adiponectin (D } \uparrow) \\
\text { Ghrelin (D } \downarrow \text { ) }\end{array}$ & [77] \\
\hline & Puerarin & $\begin{array}{l}\text { Sprague-Dawley rats. } \\
\text { HFD-feeding ( } 4 \text { weeks) }\end{array}$ & & $\uparrow$ Leptin (P $\downarrow$ ) & $\begin{array}{l}\uparrow \text { Steatosis (P } \downarrow \text { ) } \\
\uparrow \text { Inflammation (P } \downarrow \text { ) } \\
\uparrow T G \text {, cholesterol }(\mathrm{P} \downarrow \text { ) } \\
\downarrow \text { Leptin receptor (P } \uparrow \text { ) } \\
\downarrow \text { pJAK2, pSTAT (P } \uparrow \text { ) }\end{array}$ & {$[78,79]$} \\
\hline & $\begin{array}{l}\text { Puerarin } 900 \mathrm{mg} / \mathrm{Kg} \text { b.w./day } \\
\text { (2 weeks) }\end{array}$ & $\begin{array}{l}\text { C57BL/6J mice. MCD- } \\
\text { feeding ( } 2 \text { weeks) }\end{array}$ & & $\begin{array}{l}--\mathrm{TG} \text {, cholesterol (P } \downarrow \text { ) } \\
\downarrow \text { HDL (P--) } \\
\uparrow \mathrm{LDL}(\mathrm{P} \downarrow) \\
\uparrow T N F \alpha(\mathrm{P} \downarrow) \\
\uparrow \mathrm{IL}-6(\mathrm{P}--)\end{array}$ & $\begin{array}{l}\uparrow \mathrm{NAS}(\mathrm{P} \downarrow) \\
\downarrow \mathrm{PPAR} \gamma(\mathrm{P} \uparrow) \\
\uparrow \mathrm{NF}-\mathrm{kB}(\mathrm{P}--)\end{array}$ & [80] \\
\hline & $\begin{array}{l}\text { Genistein } 1 \mathrm{mg} / \mathrm{Kg} \text { b.w./day } \\
\text { (60 days) }\end{array}$ & $\begin{array}{l}\text { Wistar rats. High fructose } \\
\text { diet-feeding ( } 60 \text { days) }\end{array}$ & $\begin{array}{l}\uparrow \text { Body weight }(\mathrm{G} \downarrow) \\
\uparrow \text { Liver weight }(\mathrm{G} \downarrow)\end{array}$ & $\begin{array}{l}\uparrow A S T, \text { ALT, } \gamma \mathrm{GT}, \mathrm{LDH}(\mathrm{G} \downarrow) \\
\uparrow \mathrm{TG}, \mathrm{FFA} \text {, cholesterol }(\mathrm{G} \downarrow) \\
\uparrow \text { Phospholipids }(\mathrm{G} \downarrow) \\
\uparrow \mathrm{LDL}, \text { VLDL }(\mathrm{G} \downarrow) \\
\downarrow \text { HDL }(\mathrm{G} \uparrow) \\
\uparrow \mathrm{Glucose} \text {, insulin }(\mathrm{G} \downarrow) \\
\uparrow \mathrm{HOMA}-\mathrm{IR}(\mathrm{G} \downarrow) \\
\uparrow \mathrm{TNF}, \mathrm{IL}-6(\mathrm{G} \downarrow) \\
\uparrow \mathrm{TBARS}(\mathrm{G} \downarrow) \\
\downarrow \text { Vit C, Vit E, GSH }(\mathrm{G} \uparrow) \\
\downarrow \text { Nitrite }(\mathrm{G} \uparrow) \\
\uparrow \text { Nitrosothiol }(\mathrm{G} \downarrow)\end{array}$ & $\begin{array}{l}\uparrow N A S(G \downarrow) \\
\uparrow L D H(G \downarrow) \\
\uparrow T G, \text { FFA, cholesterol }(\mathrm{G} \downarrow) \\
\downarrow \text { Phospholipids }(\mathrm{G} \uparrow) \\
\uparrow \text { TBARS(G }) \\
\uparrow 3-\mathrm{NT}(\mathrm{G} \downarrow) \\
\downarrow \text { Nitrite }(\mathrm{G} \uparrow) \\
\uparrow \text { Nitrosothiol }(\mathrm{G} \downarrow) \\
\downarrow \text { GPx, GR, Vit } \mathrm{C}, \text { Vit E, GSH }(\mathrm{G} \uparrow)\end{array}$ & [81] \\
\hline & $\begin{array}{l}\text { Genistein } 1,2 \text { and } 4 \mathrm{~g} / \mathrm{Kg} \text { food } \\
\text { (12 weeks) }\end{array}$ & $\begin{array}{l}\text { C57BL/6J mice. HFD- } \\
\text { feeding (12 weeks) }\end{array}$ & $\begin{array}{l}\uparrow \text { Body weight }(\mathrm{G} \downarrow) \\
\uparrow \text { Fat pads }(\mathrm{G} \downarrow)\end{array}$ & $\begin{array}{l}\uparrow \mathrm{ALT}(\mathrm{G} \downarrow) \\
--\mathrm{AST}(\mathrm{G}--) \\
\downarrow \mathrm{TG}(\mathrm{G} \downarrow) \\
\uparrow \mathrm{FFA}, \text { cholesterol }(\mathrm{G} \downarrow) \\
\uparrow \mathrm{LDL}(\mathrm{G} \downarrow) \\
\downarrow \mathrm{HDL}(\mathrm{G} \uparrow)\end{array}$ & $\begin{array}{l}\uparrow N A S(G \downarrow) \\
\uparrow T G, \text { cholesterol }(\mathrm{G} \downarrow)\end{array}$ & [82] \\
\hline
\end{tabular}




\begin{tabular}{|c|c|c|c|c|c|c|}
\hline 童 & $\begin{array}{l}\text { Cy-3-g } 1 \mathrm{~g} / \mathrm{Kg} \text { food (12 } \\
\text { weeks) }\end{array}$ & KKAy mice & $\begin{array}{l}\text { Body weight }(\mathrm{Cy}-3-\mathrm{g} \downarrow) \\
\text { Liver weight (Cy-3-g } \mathrm{g} \downarrow) \\
\text { Fat pads }(\mathrm{Cy}-3-\mathrm{g} \downarrow)\end{array}$ & $\begin{array}{l}\text { Cholesterol (Cy-3-g--) } \\
\text { LDL, HDL (Cy-3-g--) } \\
\text { TG (Cy-3-g } \downarrow \text { ) } \\
\text { LPL (Cy-3-g } 1 \text { ) }\end{array}$ & $\begin{array}{l}\text { Steatosis (Cy-3-g } \downarrow) \\
\text { TG (Cy-3-g } \downarrow \text { ) }\end{array}$ & [84] \\
\hline 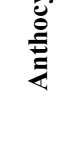 & $\begin{array}{l}\text { Cy-3-g } 100 \mathrm{mg} / \mathrm{Kg} \text { food (12 } \\
\text { weeks) }\end{array}$ & KKAy mice & & $\begin{array}{l}\text { TG (Cy-3-g } \downarrow) \\
\text { Glucose (Cy-3-g --) }\end{array}$ & $\begin{array}{l}\uparrow \text { Steatosis (Cy-3-g } \downarrow) \\
\uparrow \mathrm{TG}(\mathrm{Cy}-3-\mathrm{g} \downarrow) \\
--\mathrm{pPKC \zeta} / \mathrm{PKC \zeta}(\mathrm{Cy}-3-\mathrm{g} \uparrow) \\
\uparrow \mathrm{mtGPAT1}(\mathrm{Cy}-3-\mathrm{g} \downarrow)\end{array}$ & [85] \\
\hline
\end{tabular}

3-NT, 3-nitrotyrosine; 4-HNE, 4-hydroxy-2'-nonenal; 8-OHdG, 8-hydroxy-2'-deoxyguanosine; ALT, alanine aminotransferase; AST, aspartate aminotransferase; C, chrysin; CAT, catalase; ChREBP, carbohydrate responsive element binding protein; COX-2, cyclooxygenase-2; Cy-3-g, cyanidin-3-O--3-glucoside; CYP, cytochrome P450; D, daidzein; EGCG, (-)-epigallocatechin-3-gallate; FFA, free fatty acid; FOXO1, forkhead box protein O1; G, genistein; GPx, glutathione peroxidase; GR, glutathione reductase; GSH, glutathione; HDL, high density lipoprotein; HFD, high fat diet; HOMA-IR, homeostasis model assessment for insulin resistance; IL, interleukin; iNOS, inducible nitric oxide synthase; JAK2, janus kinase 2; JNK, c-Jun terminal kinase; LDH, lactate dehydrogenase; LDL, low density lipoprotein; LPL, lipoprotein lipase; LXRß, liver $\mathrm{X}$ receptor beta; MCP-1, monocyte chemoattractant protein-1; MDA, malondialdehyde; MMP, matrix metalloproteinase; mtGPAT1, glycerol-3-phosphate acyltransferase 1 mitochondrial; N, naringenin; NAS, NAFLD activity score; NF-KB, nuclear factor kappa B; P, puerarin; PAI-1, plasminogen activator inhibitor-1; PI3K, phosphatidylinositol 3-kinase; PKCъ, protein kinase C zeta; PPAR $\gamma$, peroxisome proliferatoractivated receptor gamma; SOD, superoxide dismutase; STAT, signal transducer and activator of transcription; TBARS, thiobarbituric acid-reactive substance; TG, triglyceride; TGF, transforming growth factor; TIMP, tissue inhibitor of metalloproteinases; $\mathbf{T N F} \boldsymbol{\alpha}$, tumor necrosis factor alpha; VLDL, very low density lipoprotein; $\boldsymbol{\alpha}$-SMA, alpha-smooth muscle actin; $\gamma \mathbf{G T}$, gamma glutamyl transferase; $\uparrow$, increase; $\downarrow$, decrease; - , no effect. 
Table 3. Effects of flavonoids on in vitro models of NAFLD.

\begin{tabular}{|c|c|c|c|c|c|}
\hline Group & Flavonoid treatment & Model & & Effects & Ref. \\
\hline \multirow{8}{*}{$\frac{n}{\grave{O}}$} & Quercetin $0.1-100 \mu \mathrm{M}(48 \mathrm{~h})$ & HepG2. FFA $1 \mathrm{mM}$, insulin $50 \mathrm{nM}(48 \mathrm{~h})$ & $\begin{array}{l}\mathrm{Q} \downarrow \text { cell proliferation at } 50 \text { and } 100 \mu \mathrm{M} \\
\uparrow \mathrm{Lipid} \text { accumulation }(\mathrm{Q} \downarrow) \\
\uparrow \mathrm{TG}(\mathrm{Q} \downarrow) \\
\text {--IR } \beta \text {, IRS1 (Q--) }\end{array}$ & $\begin{array}{l}\downarrow \mathrm{pIR} \beta, \text { pIRS1 }(\mathrm{Q} \uparrow) \\
\uparrow \mathrm{SREBP}-1 \mathrm{c}, \mathrm{FAS}(\mathrm{Q} \downarrow) \\
\text {--PPAR } \alpha, \text { CPT1, MTP, FABP1 (Q--) }\end{array}$ & [27] \\
\hline & Quercetin $10 \mu \mathrm{M}(24 \mathrm{~h})$ & HepG2. Oleic acid 2 mM (24h) & $\begin{array}{l}\downarrow \text { cell proliferation }(\mathrm{Q} \uparrow) \\
\uparrow \mathrm{ALT}(\mathrm{Q} \downarrow) \\
\uparrow \mathrm{TG}(\mathrm{Q} \downarrow) \\
\downarrow \text { Glucose uptake, urea, albumin }(\mathrm{Q} \uparrow) \\
\uparrow \mathrm{MDA}(\mathrm{Q} \downarrow)\end{array}$ & $\begin{array}{l}\uparrow \mathrm{DNA} \text { fragmentation }(\mathrm{Q} \downarrow) \\
\uparrow \mathrm{TNF} \alpha, \mathrm{IL}-18(\mathrm{Q} \downarrow) \\
\downarrow \mathrm{GSH}(\mathrm{Q} \uparrow) \\
\uparrow \mathrm{GSSG}(\mathrm{Q} \downarrow) \\
\downarrow \mathrm{CAT}, \mathrm{GPx}, \mathrm{SOD}(\mathrm{Q} \uparrow)\end{array}$ & {$[28]$} \\
\hline & Quercetin 1-50 $\mu \mathrm{M}(24 \mathrm{~h})$ & HepG2. Oleic acid $1 \mathrm{mM}(24 \mathrm{~h})$ & $\begin{array}{l}\uparrow R O S(Q \downarrow) \\
\uparrow T G(Q \downarrow)\end{array}$ & & [33] \\
\hline & Rutin $25-200 \mu \mathrm{M}(24 \mathrm{~h})$ & HepG2. Oleic acid $1 \mathrm{mM}(24 \mathrm{~h})$ & $\begin{array}{l}\uparrow R O S(R \downarrow) \\
\uparrow T G(R \downarrow)\end{array}$ & & {$[33]$} \\
\hline & Kaempferol 50-150 $\mu \mathrm{M}(24 \mathrm{~h})$ & HepG2. Oleic acid $1 \mathrm{mM}(24 \mathrm{~h})$ & $\begin{array}{l}\uparrow R O S(\text { Kaempferol } \downarrow) \\
\uparrow T G(\text { Kaempferol } \downarrow)\end{array}$ & & {$[33]$} \\
\hline & $\begin{array}{l}\text { Isorhamnetin 10-150 } \mu \mathrm{M} \\
\text { (24h) }\end{array}$ & HepG2. Oleic acid $1 \mathrm{mM}(24 \mathrm{~h})$ & $\begin{array}{l}\uparrow R O S \text { (Isorhamnetin } \downarrow \text { ) } \\
\uparrow T G \text { (Isorhamnetin } \downarrow \text { ) }\end{array}$ & & {$[33]$} \\
\hline & Myricetin $10-150 \mu \mathrm{M}$ (24h) & HepG2. Oleic acid $1 \mathrm{mM}(24 \mathrm{~h})$ & $\begin{array}{l}\uparrow R O S(M y \downarrow) \\
\uparrow T G(M y \downarrow)\end{array}$ & & {$[33]$} \\
\hline & Galangin $1-40 \mu \mathrm{M}(24 \mathrm{~h})$ & HepG2. Oleic acid $1 \mathrm{mM}(24 \mathrm{~h})$ & $\begin{array}{l}\uparrow R O S \text { (Galangin } \downarrow \text { ) } \\
\uparrow T G(\text { Galangin --) }\end{array}$ & & {$[33]$} \\
\hline \multirow{3}{*}{ 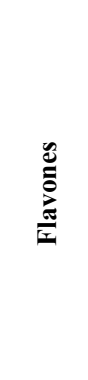 } & Chrysin 1-15 $\mu \mathrm{M}(24 \mathrm{~h})$ & HepG2. Oleic acid $1 \mathrm{mM}(24 \mathrm{~h})$ & $\begin{array}{l}\uparrow \mathrm{ROS}(\mathrm{C} \uparrow) \\
\uparrow \mathrm{TG}(\mathrm{C}--)\end{array}$ & & {$[33]$} \\
\hline & Apigenin 10-125 $\mu \mathrm{M}(24 \mathrm{~h})$ & HepG2. Oleic acid $1 \mathrm{mM}(24 \mathrm{~h})$ & $\begin{array}{l}\uparrow R O S \text { (Apigenin } \uparrow \text { ) } \\
\uparrow T G \text { (Apigenin --) }\end{array}$ & & {$[33]$} \\
\hline & Luteolin 10 and $20 \mu \mathrm{M}(24 \mathrm{~h})$ & HepG2. Palmitate $0.4 \mathrm{mM}$ (24h) & $\begin{array}{l}\uparrow \text { ROS (Luteolin } \downarrow \text { ) } \\
\uparrow \text { Lipid accumulation (Luteolin } \downarrow \text { ) } \\
\uparrow \text { TG (Luteolin } \downarrow \text { ) } \\
\text {--pAMPK } \alpha, \text { pACC (Luteolin } \uparrow \text { ) } \\
\uparrow \text { SREBP-1c, FAS (Luteolin } \downarrow \text { ) } \\
\uparrow \text { CPT1 (Luteolin } \uparrow \text { ) }\end{array}$ & & {$[63]$} \\
\hline
\end{tabular}




\begin{tabular}{|c|c|c|c|c|c|}
\hline & Luteolin $1-15 \mu \mathrm{M}(24 \mathrm{~h})$ & HepG2. Oleic acid $1 \mathrm{mM}(24 \mathrm{~h})$ & $\begin{array}{l}\uparrow R O S \text { (Luteolin } \downarrow \text { ) } \\
\uparrow T G \text { (Luteolin } \downarrow \text { ) }\end{array}$ & & [33] \\
\hline \multirow{3}{*}{ 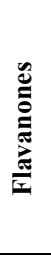 } & Naringenin $10-150 \mu \mathrm{M}(24 \mathrm{~h})$ & HepG2. Oleic acid $1 \mathrm{mM}(24 \mathrm{~h})$ & $\begin{array}{l}\uparrow \operatorname{ROS}(\mathrm{N} \uparrow) \\
\uparrow T \mathrm{~T}(\mathrm{~N}--) \\
\end{array}$ & & [33] \\
\hline & Hesperetin 5-75 $\mu \mathrm{M}(24 \mathrm{~h})$ & HepG2. Oleic acid $1 \mathrm{mM}(24 \mathrm{~h})$ & $\begin{array}{l}\uparrow R O S \text { (Hesperetin } \downarrow \text { ) } \\
\uparrow T G \text { (Hesperetin --) }\end{array}$ & & [33] \\
\hline & Taxifolin $10-150 \mu \mathrm{M}(24 \mathrm{~h})$ & HepG2. Oleic acid $1 \mathrm{mM}(24 \mathrm{~h})$ & $\begin{array}{l}\uparrow R O S \text { (Taxifolin } \downarrow \text { ) } \\
\uparrow \text { TG (Taxifolin } \downarrow \text { ) }\end{array}$ & & [33] \\
\hline \multirow{4}{*}{ 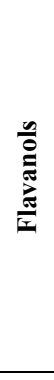 } & EGCG $10-150 \mu \mathrm{M}(24 \mathrm{~h})$ & HepG2. Oleic acid $1 \mathrm{mM}(24 \mathrm{~h})$ & $\begin{array}{l}\uparrow \operatorname{ROS}(\text { EGCG } \downarrow \text { ) } \\
\uparrow T G(E G C G \downarrow)\end{array}$ & & [33] \\
\hline & $(+)$-Catechin $25-200 \mu \mathrm{M}(24 \mathrm{~h})$ & HepG2. Oleic acid $1 \mathrm{mM}(24 \mathrm{~h})$ & $\begin{array}{l}\uparrow \operatorname{ROS}((+) \text {-Catechin } \downarrow) \\
\uparrow \operatorname{TG}((+) \text {-Catechin } \downarrow)\end{array}$ & & [33] \\
\hline & $\begin{array}{l}\text { (-)-Epicatechin } 50-200 \mu \mathrm{M} \\
(24 \mathrm{~h})\end{array}$ & HepG2. Oleic acid $1 \mathrm{mM}(24 \mathrm{~h})$ & $\begin{array}{l}\uparrow \operatorname{ROS}((-) \text {-Epicatechin } \downarrow) \\
\uparrow \mathrm{TG}((-) \text {-Epicatechin } \downarrow)\end{array}$ & & [33] \\
\hline & $\begin{array}{l}\text { Procyanidin B1 10, } 20 \text { and } 30 \\
\mu \mathrm{M}(48 \mathrm{~h})\end{array}$ & HepG2. Palmitic acid $0.5 \mathrm{mM}(48 \mathrm{~h})$ & 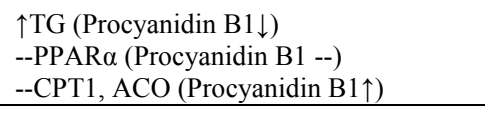 & & {$[74]$} \\
\hline \multirow{2}{*}{ 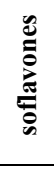 } & Daidzein $10-150 \mu \mathrm{M}(24 \mathrm{~h})$ & HepG2. Oleic acid $1 \mathrm{mM}(24 \mathrm{~h})$ & $\begin{array}{l}\uparrow \operatorname{ROS}(\mathrm{D} \downarrow) \\
\uparrow \mathrm{TG}(\mathrm{D} \downarrow)\end{array}$ & & [33] \\
\hline & Genistein $1-15 \mu \mathrm{M}(24 \mathrm{~h})$ & HepG2. Oleic acid $1 \mathrm{mM}(24 \mathrm{~h})$ & $\begin{array}{l}\uparrow R O S(G \downarrow) \\
\uparrow T G(G--)\end{array}$ & & [33] \\
\hline 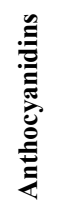 & $\begin{array}{l}\text { Cy-3-g } 50 \mu \mathrm{M}(2 \mathrm{~h} \text { before } \\
\text { glucose treatment) }\end{array}$ & $\begin{array}{l}\text { Hepatocytes from C57BL/6J mice fed a } \\
\text { HFD. Glucose } 35 \mathrm{mM}(12 \mathrm{~h})\end{array}$ & $\begin{array}{l}\downarrow \text { Cell viability }(\mathrm{Cy}-3 \text { - } \mathrm{g} \uparrow) \\
\uparrow \mathrm{LDH}(\mathrm{Cy}-3 \text { - } \mathrm{g} \text { ) } \\
\uparrow \text { ROS, carbonyl groups (Cy-3-g } \downarrow \text { ) } \\
\uparrow \text { Apoptosis (Cy-3-g } \downarrow \text { ) } \\
\uparrow \text { Procaspase-3, procaspase-8, procaspase- } \\
9 \text {, PARP cleavage (Cy-3-g } \downarrow \text { ) }\end{array}$ & $\begin{array}{l}\uparrow \text { Caspase-3, caspase-9 activities }(C y-3-\mathrm{g} \downarrow) \\
\text {--Caspase-8 activity (Cy-3-g--) } \\
\downarrow \text { pAKT }(\text { Cy-3-g } \uparrow) \\
\uparrow \text { pJNK (Cy-3-g } \downarrow \text { ) }\end{array}$ & [86] \\
\hline
\end{tabular}

ACC, acetyl-CoA carboxylase; ACO, acyl-CoA oxidase; ALT, alanine aminotransferase; AMPK, AMP-activated protein kinase; C, chrysin; CAT, catalase; CPT1, carnitine palmitoyltransferase 1; Cy-3-g, cyanidin3-O-ß-glucoside; D, daidzein; EGCG, (-)-epigallocatechin-3-gallate; FABP, fatty acid binding protein; FAS, fatty acid synthase; FFA, free fatty acid; G, genistein; GPx, glutathione peroxidase; GSH, glutathione; GSSG, oxidized glutathione; HFD, high fat diet; IL, interleukin; IRS1, insulin receptor substrate 1; IRß, insulin receptor beta; JNK, c-Jun terminal kinase; LDH, lactate dehydrogenase; MDA, malondialdehyde; MTP, microsomal triglyceride transfer protein; My, myricetin; N, naringenin; PARP, poly (ADP-ribose) polymerase; PPARa, peroxisome proliferator-activated receptor alpha; $\mathbf{Q}$, quercetin; R, rutin; ROS, reactive oxygen species; SOD, superoxide dismutase; SREBP, sterol regulatory element-binding protein; TG, triglyceride; TNF $\boldsymbol{\alpha}$, tumor necrosis factor alpha; $\uparrow$, increase; $\downarrow$, decrease; --, no effect. 


\section{OTHER NATURAL COMPOUNDS}

Silybum marianum, known as milk thistle, is a member of Asteraceae family that contains a mixture of flavonolignans named silymarin and two flavonoids (quercetin and taxifolin). Silymarin is a lipophilic extract composed of silybin A, silybin B, isosilybin A, isosilybin B, silychristin, silydianin and isosilychristin. Silibinin, a mixture of silybin A and silybin B, is the major constituent of silymarin. This mixture of flavonolignans displays a lipid-lowering effect in both in vitro and in vivo models of induced lipid accumulation [28, 89, 90]. It has been proven that silibinin administration counteracts the progression of liver injury in several nutritional models of NASH, by improving antioxidant status, insulin resistance, steatosis, ballooning and inflammation. This effect appears to be a consequence of the capacity of silibinin to reduce increased NF- $\mathrm{KB}$ activity and subsequent iNOS overexpression as well as ROS and reactive nitrogen species (RNS) levels. Moreover, the increased phosphorylation of JNK, which is associated with insulin resistance and inflammation, is attenuated by silibinin. Likewise, silbinin diminishes DNA damage and lipoperoxidation and increases SCD1 and FABP1 expressions, that are downregulated in NASH models [91-93]. SCD1 and FABP1 downregulation is associated with cell sensitization to saturated fatty acid-induced death [94] and lipotoxicity [95], respectively. Silymarin also displays antifibrogenic effects through the downregulation of TGF $\beta$ [96], one of the critical cytokines involved in fibrogenesis, and the suppression of hepatic stellate cells activation and $\alpha_{1}$-procollagen production, although it can not completely block the histological appearance of liver fibrosis. Moreover, the increase in nuclear translocation of $\mathrm{Nrf}$, that may upregulate many antioxidant genes, might contribute to the antioxidant capacity of silymarin besides the reduction of TNF $\alpha$ expression, probably by reversing ERK activation [97]. On the contrary, non-effect of silymarin has been shown on phosphorylation levels of ERK, showing in addition an anti-apoptotic activity by lowering activation of procaspase-3 to active caspase-3 [96]. Silymarin has also been reported to display positive effects in patients with NAFLD $[98,99]$, in which the efficacy of this extract may be more readily observed compared with patients with hepatitis $\mathrm{C}$, because of their higher plasma flavonolignan concentrations and more extensive enterohepatic cycling [100]. Combination of silibinin with phospholipids appears to increase its bioavailability [101]. Several clinical studies evidence the efficacy of silibinin combined with phospholipids and vitamin E in patients with NAFLD, who exhibit improvements in aspartate aminotransferase (AST), alanine aminotransferase (ALT) and gamma glutamyl transferase ( $\gamma \mathrm{GT}$ ) levels, insulin resistance and liver histology in addition to the normalization of the cholestasis and cholesterol levels [102-105].

Curcumin is a polyphenol derived from Curcuma longa (turmeric), a food spice and colouring agent widely utilized in Indian and other South Asia countries. Its use in in vivo models of NASH reveals an improvement of steatosis, inflammation, ballooning and fibrosis, and a reduction of blood serum monocyte chemoattractant protein (MCP)-1, TNF $\alpha$ and IL-6 levels. Lipoperoxidation is attenuated, the antioxidant status is enhanced, and the expressions of NF- $\mathrm{kB}$, suppressor of cytokine signaling 3 (SOCS3), SREBP-1c, FAS, ACC and 3-hydroxy-3-methylglutaryl (HMG)-CoA reductase are decreased in the liver after curcumin treatment $[80,106,107]$. The beneficial effects of curcumin on steatosis and inflammation could be mediated by the STAT3 signaling activation and the improvement of 
mitochondrial function [107]. In vitro studies show that curcumin is able to reduce induced lipid accumulation by downregulating lipogenic proteins and upregulating the expression of fatty acid $\beta$ oxidation-related genes as PPAR $\alpha$, via AMPK phosphorylation [108]. Moreover, this polyphenol seems to protect hepatocytes from lipoapoptosis [109] and iron-induced insulin resistance and oxidative stress in cells treated with stearic acid, probably reducing phosphorylated JNK levels [110].

Resveratrol (trans-3,4',5-trihydroxystilbene) is a polyphenol abundantly present in red wine and, to a lesser extent, in other foods such as grapes or nuts. This compound reduces body weight in obese mice, enhances antioxidant enzymes levels and diminishes lipoperoxidation, improving also serum lipid profile and hepatic steatosis through the increase of SIRT1 expression and phosphorylation levels of AMPK, and FOXO1, and promoting FOXO1 translocation from the nucleus to the cytoplasm [111]. Hepatoprotective actions of resveratrol and the mechanisms responsible of these beneficial effects, observed in in vitro and in vivo studies, have been recently reviewed [112]. A polyphenol extract also obtained from red wine is Provinol $^{\mathrm{TM}}$, that contains proanthocyanidols, prodelphinidol, anthocyanins, catechin, resveratrol and others. This extract could help to prevent steatosis through SIRT1upregulation and subsequent AMPK and PPAR $\gamma$ coactivador (PGC) $1 \alpha$ increased expressions, leading to an enhanced fatty acid $\beta$-oxidation [113]. On the other hand, wine stored in oak barrels improves its organoleptic properties, including better taste. Indeed, many bioactives polyphenols contained in red wine derive from oak during the maturation. Oak bark extract treatment in a NAFLD model in rats displays multiple benefits, such as the reduction of body weight and abdominal fat pads, the improvement of insulin resistance, serum lipid profile and liver histology, as well as the recovery of cardiovascular structure and function [114].

Theaflavin is the major polyphenol derived from black tea, the most widely consumed tea in the world. Its use in a mouse model of fatty liver resulted in a decrease of hepatic steatosis, oxidative stress, inflammation and apoptosis, contributing to its protective effect against ischemia-reperfusion injury [115]. Green tea contents a high amount of catechins as EGCG, whose beneficial properties on NAFLD have been summarized herein. Several studies in murine models show the protective effect of green tea against induced non-alcoholic steatohepatitis [116, 117]. Likewise it has been reported an improved liver function and fatty liver status in patients with NAFLD after consuming green tea with high-density catechins for 12 weeks [118]. Previously, it had been published a further review about therapeutic potential of green tea and their catechins in this disease, based on studies carried out in in vitro and animal models. The mechanisms of action of green tea against NAFLD could implicate the prevention of steatosis through the reduction of intestinal lipid and carbohydrate absorption and the decrease of adipose lipolysis, in addition to the reduction of de novo lipogenesis and the enhancement of fatty acid oxidation and thermogenesis, as well as the improvement of insulin sensitivity. Moreover, antioxidant and antiinflammatory activities could contribute to prevent the progression from simple steatosis to steatohepatitis [119].

The effects of various black soybean powder concentrations have been investigated in a model of NAFLD consisting of high cholesterol and HFD-fed mice. Black soybean, that presents a high content of isoflavones, was able to reduce body, liver and abdominal and epididymal adipose tissue weights gain in 
a dose-dependent manner. Black soybean supplementation also reduced the levels of total cholesterol and triglyceride in the liver, with the subsequent decrease in the lipid droplets size. This cholesterol- and triglyceride-lowering effect was due, at least in part, to the reduction of the expression of SREBP-2, HMG-CoA reductase, PPAR $\alpha$ and PPAR $\gamma$ as well as the increase of ABCA1 expression. The reduction in free fatty acids, glucose and insulin blood levels observed after black soybean treatment was the result of the increase of serum adiponectine levels, stimulating AMPK activation and improving insulin resistance. The improvement in cholesterol accumulation and insulin resistance, besides the antioxidant and antilipoperoxidative activities of black soybean, confer a protective capacity against NAFLD [120]. On the contrary, other studies have reported an induction of PPAR $\alpha$, in addition to ACO expressions by soybean, thereby diminishing lipid accumulation through the enhancement of fatty acid $\beta$-oxidation [121, 122].

There are many other species of plants proven to present preventive effects on NAFLD. Thus, Taraxacum officinale (dandelion) shows properties similar to black soybean, as it has been observed in murine models of NAFLD a significant reduction of hepatic lipid accumulation, body and liver weights and serum cholesterol, triglyceride, insulin and glucose levels, with an improved insulin resistance by AMPK pathway activation, after dandelion leaf extract treatment [123, 124]. Myrcia bella is a species belonging to the Myrtaceae family whose principle constituents are phenolic acids and flavonoids, such as quercetin and myricetin. The extract of leaves of this species presents hypoglycemic activity, probably due to the activation of the insulin signaling pathway involving PI3K/AKT proteins that leads in turn to the store and prevention of the degradation of glucose in the liver. Moreover, the extract can act as a hypolipidemic agent by reducing the levels of serum lipids [125]. In the same way, the effect of flavonoids obtained from Rosa laevigata Michx fruit, mainly rutin, quercetin, kaempferol, luteolin, apigenin and liquiritigenin, have been studied in a model of HFD-induced NAFLD in rats. It was observed a lower weight gain and a hepatoprotective effect showing a dose-dependent reduction of serum AST and ALT as well as blood lipid and glucose levels after flavonoids treatment. It was also shown a significant decrease in lipid peroxidation and CYP2E1 expression due to the antioxidant capacity of flavonoids. Expression of SREBP-1c, FASN, SCD1, PPAR $\gamma$, long-chain fatty acyl-CoA ligase (ACSL) 5 and ACC was reduced and levels of pAMPK $\alpha$, ACO1 and PPAR $\alpha$ were increased in flavonoids-treated rats, reverting the effect of HFD. This led to a fatty acid synthesis downregulation and a fatty acid $\beta$ oxidation promotion with a subsequent lipid content reduction in hepatocytes [90]. Phyllanthus urinaria, a traditional medicinal plant, with a high amount of tannins and flavonoids, has also been documented to ameliorate the severity of steatohepatitis in in vitro and in vivo nutritional models. This effect seems to be attributed to the suppression of CYP2E1-mediated oxidative stress and JNK and NFmediated inflammatory response, in addition to the induction of fatty acid oxidation through CYP4A10 upregulation and the suppression of lipogenic transcription factor C/EBP $\beta$ [126]. Likewise, Ilex hainanensis Merr., whose major bioactive components are chlorogenic acid, kaempferol-7-O- $\beta$-Dglucoside and ilexgenin A [127], displays preventive effects on hepatic induced steatosis by alleviating insulin resistance and regulating lipid metabolism, inflammation and oxidative stress, through the regulation of PPAR $\alpha$ and CYP2E1 expressions [128, 129]. The lipid-lowering effect of Ginkgo biloba, whose principle active ingredients are quercetin, kaempferol and isorhamnetin, seems to be attributed to 
the content of these flavonoids, through the CPT1A-mediated fatty acid oxidation enhancement [130, 131]. Furthermore, there are several traditional Chinese medicine formulas, as Salvia-Nelumbinis naturalis or Hugan Qingzhi tablet, with a lipid-lowering effect on induced steatosis, via enhancing hepatic insulin sensitivity and fatty acid oxidation or reducing lipogenesis $[132,133]$.

Flavangenol ${ }^{\circledR}$, one of several pine bark extract products, is also reported to exert a lipid-lowering action in the liver of mice with insulin resistance, abnormalities of carbohydrate and lipid metabolism, hypertension and a marked fatty liver, by increasing the expression of fatty acid oxidation-related genes. The effect of Flavangenol ${ }^{\circledR}$ could be attributed in part to procyanidin B1, its major bioactive component [74]. Other authors explain the beneficial effects of total flavonoids from citrus on NASH in mice fed with a HFD by the modulation of the SIRT1/PGC-1 $\alpha$ signal pathway, which might in turn be related to the improvement of oxidative stress and lipid peroxidation [134]. Polyphenols from pomegranate flowers seem to improve also insulin resistance and steatosis in rats with diabetes and non-alcoholic fat liver disease, probably by enhancing PON1 expression in the liver [135]. In the same way, polyphenolic extracts obtained from blueberries [136, 137], mulberries [137] and bayberries [138] show preventive effects on NAFLD, inhibiting body weight gain, decreasing serum cholesterol, reducing insulin resistance and attenuating lipid accumulation, as well as improving plasma antioxidant status and inhibiting the inflammatory and apoptotic responses. Likewise, polyphenolic extract from lotus root, the edible rhizome of Nelumbo nucifera, rich in proanthocyanidins, alleviates hepatic steatosis by suppressing lipogenic enzymes activities [139].

\section{CONCLUSION}

Polyphenols, as flavonoids and many other related natural compounds, display beneficial effects on NAFLD, preventing multiple disorders associated with the disease. These molecules possess the ability to modulate the expression of several genes whose deregulation contributes to lipid accumulation, fibrosis, inflammation, oxidative stress or insulin resistance. Their lipid-lowering effects seem to be associated with their capacity to regulate the expression of genes mainly involved in de novo lipogenesis and fatty acid $\beta$-oxidation, although the antioxidant and anti-inflammatory properties of these compounds might also contribute to this capacity (Fig. 3). Overall, in view of results obtained in in vitro and in vivo models, as well as in patients with NAFLD, it may be concluded that the use of flavonoids and other natural substances should be considered as therapy for preventing NAFLD progression. 


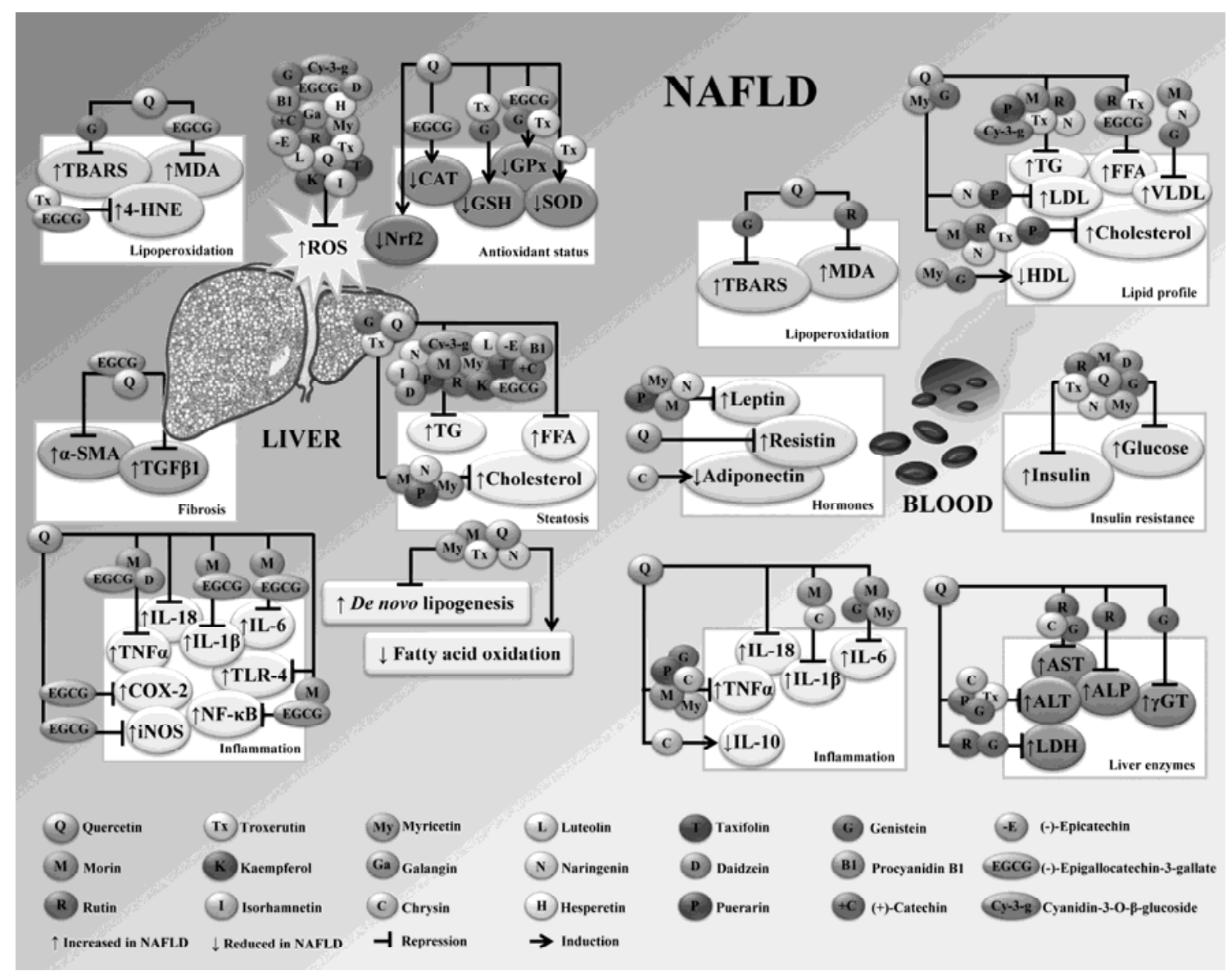

Fig (3). Overview of the mechanisms of action reported for different flavonoids. Most studied flavonoids are able to counteract blood parameter alterations and liver disorders associated with NAFLD progression. Lipid peroxidation is reduced, and antioxidant status enhanced, leading to a reduction of reactive oxygen species (ROS). Fibrosis and inflammation are ameliorated and steatosis is diminished, fundamentally through a reduction of triglyceride (TG) levels, probably mediated by modulation of de novo lipogenesis- and fatty acid oxidation-related gene expression, altered in NAFLD. Moreover, there is an improvement of insulin resistance, serum lipid profile and liver function, as shown by the normalization in the levels of liver enzymes.

\section{CONFLICT OF INTEREST STATEMENT}

All authors state no financial and personal relationships with other people or organizations that could inappropriately influence this work.

\section{ACKNOWLEDGEMENTS}

This work was supported by grants to Javier González-Gallego and Sonia Sánchez Campos from Ministerio de Economía y Competitividad/FEDER (BFU2013-48141-R) and Javier González Gallego and José Luis Olcoz from Junta de Castilla y León (LE135U13 and GRS1000/A/14, respectively). María V. García-Mediavilla was supported by CIBERehd contract. CIBERehd is funded by the Instituto de Salud Carlos III, Spain. 


\section{ABBREVIATIONS}

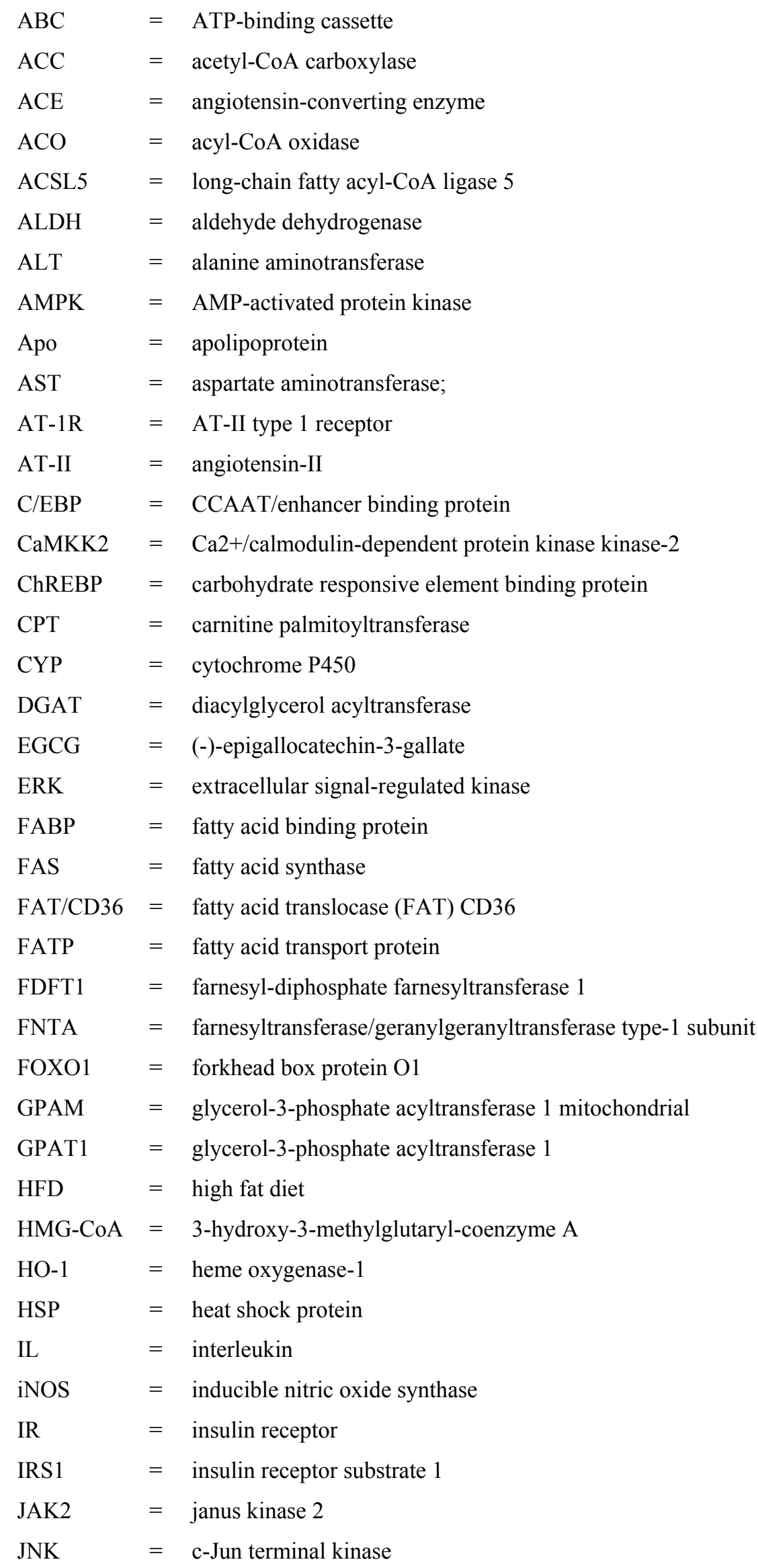




$$
\begin{aligned}
& \mathrm{LPL}=\text { lipoprotein lipase } \\
& \mathrm{MCAD}=\text { medium-chain acyl-CoA dehydrogenase } \\
& \text { MCP-1 = monocyte chemoattractant protein-1 } \\
& \text { mTOR }=\text { mammalian target of rapamycin } \\
& \text { MTP }=\text { microsomal triglyceride transfer protein } \\
& \text { NAFLD }=\text { non-alcoholic fatty liver disease } \\
& \text { NAMPT }=\text { nicotinamide phosphoribosyltransferase } \\
& \mathrm{NASH}=\text { non-alcoholic steatohepatitis } \\
& \mathrm{NF}-\kappa \mathrm{B}=\text { nuclear factor kappa } \mathrm{B} \\
& \text { NLRP3 }=\text { NOD-like receptor family pyrin domain-containing } 3 \\
& \text { Nrf2 }=\text { nuclear factor (erythroid-derived 2)-related factor-2 } \\
& \text { PARP1 = poly (ADP-ribose) polymerase-1 } \\
& \text { PGC1 } \alpha=\text { PPAR } \gamma \text { coactivador-1 alpha } \\
& \mathrm{PI} 3 \mathrm{~K}=\text { phosphatidylinositol 3-kinase } \\
& \text { PON1 }=\text { paraoxonase } 1 \\
& \text { PPAR }=\text { peroxisome proliferator activated receptor } \\
& \text { RNS }=\text { reactive nitrogen species } \\
& \text { ROS }=\text { reactive oxygen species } \\
& \mathrm{RXR} \alpha=\text { retinoid } \mathrm{X} \text { receptor alpha } \\
& \mathrm{S} 1 \mathrm{P}=\text { sphingosine } 1 \text {-phosphate } \\
& \mathrm{SCD} 1=\text { stearoyl-CoA desaturase-1 } \\
& \text { SIRT1 }=\text { silent mating type information regulation } 2 \text { homolog } 1 \\
& \text { SOCS3 } 3=\text { suppressor of cytokine signaling } 3 \\
& \text { SphK1 }=\text { sphingosine kinase } 1 \\
& \text { SREBP }=\text { sterol regulatory element binding protein } \\
& \text { STAT }=\text { signal transducer and activator of transcription } 3 \\
& \mathrm{TGF}=\text { transforming growth factor } \\
& \text { TLR }=\text { toll-like receptor } \\
& \mathrm{TNF} \alpha=\text { tumor necrosis factor alpha } \\
& \text { TXNIP }=\text { thioredoxin-interacting protein } \\
& \text { VLCAD }=\text { very long-chain acyl-CoA dehydrogenase } \\
& \text { VLDL }=\text { very low density lipoprotein } \\
& \gamma \mathrm{GT} \quad=\quad \text { gamma glutamyl transferase }
\end{aligned}
$$




\section{REFERENCES}

[1] Vernon, G.; Baranova, A.; Younossi, Z. M. Systematic review: the epidemiology and natural history of non-alcoholic fatty liver disease and non-alcoholic steatohepatitis in adults. Aliment. Pharmacol. Ther. 2011, 34, 274-285.

[2] Machado, M.; Cortez-Pinto, H. Non-alcoholic steatohepatitis and metabolic syndrome. Curr. Opin. Clin. Nutr. Metab. Care 2006, 9, 637-642.

[3] Tiniakos, D. G.; Vos, M. B.; Brunt, E. M. Nonalcoholic fatty liver disease: pathology and pathogenesis. Annu. Rev. Pathol. 2010, 5, 145-171.

[4] Tilg, H.; Moschen, A. R. Evolution of inflammation in nonalcoholic fatty liver disease: the multiple parallel hits hypothesis. Hepatology 2010, 52, 1836-1846.

[5] Farrell, G. C.; Larter, C. Z. Nonalcoholic fatty liver disease: from steatosis to cirrhosis. Hepatology 2006, 43, S99-S112.

[6] Koo, S. H. Nonalcoholic fatty liver disease: molecular mechanisms for the hepatic steatosis. Clin. Mol. Hepatol. 2013, 19, 210-215.

[7] Neuschwander-Tetri, B. A. Nontriglyceride hepatic lipotoxicity: the new paradigm for the pathogenesis of NASH. Curr. Gastroenterol. Rep. 2010, 12, 49-56.

[8] Miquilena-Colina, M. E.; Lima-Cabello, E.; Sánchez-Campos, S.; García-Mediavilla, M. V.; Fernández-Bermejo, M.; Lozano-Rodríguez, T.; Vargas-Castrillón, J.; Buqué, X.; Ochoa, B.; Aspichueta, P.; González-Gallego, J.; García-Monzón, C. Hepatic fatty acid translocase CD36 upregulation is associated with insulin resistance, hyperinsulinaemia and increased steatosis in non-alcoholic steatohepatitis and chronic hepatitis C. Gut 2011, 60, 1394-1402.

[9] Lima-Cabello, E.; García-Mediavilla, M. V.; Miquilena-Colina, M. E.; Vargas-Castrillón, J.; Lozano-Rodríguez, T.; Fernández-Bermejo, M.; Olcoz, J. L.; González-Gallego, J.; GarcíaMonzón, C.; Sánchez-Campos, S. Enhanced expression of pro-inflammatory mediators and liver X-receptor-regulated lipogenic genes in non-alcoholic fatty liver disease and hepatitis C. Clin. Sci. (Lond) 2011, 120, 239-250.

[10] García-Mediavilla, M. V.; Pisonero-Vaquero, S.; Lima-Cabello, E.; Benedicto, I.; Majano, P. L.; Jorquera, F.; González-Gallego, J.; Sánchez-Campos, S. Liver X receptor alpha-mediated regulation of lipogenesis by core and NS5A proteins contributes to $\mathrm{HCV}$-induced liver steatosis and HCV replication. Lab. Invest. 2012, 92, 1191-1202.

[11] Uyeda, K.; Repa, J. J. Carbohydrate response element binding protein, ChREBP, a transcription factor coupling hepatic glucose utilization and lipid synthesis. Cell. Metab. 2006, 4, 107-110.

[12] Desvergne, B.; Wahli, W. Peroxisome proliferator-activated receptors: nuclear control of metabolism. Endocr. Rev. 1999, 20, 649-688.

[13] Hussain, M. M.; Shi, J.; Dreizen, P. Microsomal triglyceride transfer protein and its role in apoBlipoprotein assembly. J. Lipid Res. 2003, 44, 22-32.

[14] Torres, D. M.; Harrison, S. A. Diagnosis and therapy of nonalcoholic steatohepatitis. Gastroenterology 2008, 134, 1682-1698.

[15] González-Gallego, J.; García-Mediavilla, M. V.; Sánchez-Campos, S.; Tunón, M. J. Fruit polyphenols, immunity and inflammation. Br. J. Nutr. 2010, 104 Suppl 3, S15-27. 
[16] Ying, H. Z.; Liu, Y. H.; Yu, B.; Wang, Z. Y.; Zang, J. N.; Yu, C. H. Dietary quercetin ameliorates nonalcoholic steatohepatitis induced by a high-fat diet in gerbils. Food Chem. Toxicol. 2013, 52, 53-60.

[17] Pisonero-Vaquero, S.; García-Mediavilla, M. V.; Jorquera, F.; Majano, P. L.; Benet, M.; Jover, R.; González-Gallego, J.; Sánchez-Campos, S. Modulation of PI3K-LXRalpha-dependent lipogenesis mediated by oxidative/nitrosative stress contributes to inhibition of HCV replication by quercetin. Lab. Invest. 2014, 94, 262-274.

[18] Bladé, C.; Baselga-Escudero, L.; Salvadó, M. J.; Arola-Arnal, A. miRNAs, polyphenols, and chronic disease. Mol. Nutr. Food Res. 2013, 57, 58-70.

[19] Eslamparast, T.; Eghtesad, S.; Hekmatdoost, A.; Poustchi, H. Probiotics and nonalcoholic fatty liver disease. Middle East. J. Dig. Dis. 2013, 5, 129-136.

[20] Kawabata, K.; Sugiyama, Y.; Sakano, T.; Ohigashi, H. Flavonols enhanced production of antiinflammatory substance(s) by Bifidobacterium adolescentis: prebiotic actions of galangin, quercetin, and fisetin. Biofactors 2013, 39, 422-429.

[21] Marcolin, E.; San-Miguel, B.; Vallejo, D.; Tieppo, J.; Marroni, N.; González-Gallego, J.; Tuñón, M. J. Quercetin treatment ameliorates inflammation and fibrosis in mice with nonalcoholic steatohepatitis. J. Nutr. 2012, 142, 1821-1828.

[22] Crespo, I.; García-Mediavilla, M. V.; Almar, M.; González, P.; Tuñón, M. J.; Sánchez-Campos, S.; González-Gallego, J. Differential effects of dietary flavonoids on reactive oxygen and nitrogen species generation and changes in antioxidant enzyme expression induced by proinflammatory cytokines in Chang Liver cells. Food Chem. Toxicol. 2008, 46, 1555-1569.

[23] Tieppo, J.; Cuevas, M. J.; Vercelino, R.; Tuñón, M. J.; Marroni, N. P.; González-Gallego, J. Quercetin administration ameliorates pulmonary complications of cirrhosis in rats. J. Nutr. 2009, 139, 1339-1346.

[24] de David, C.; Rodrigues, G.; Bona, S.; Meurer, L.; González-Gallego, J.; Tuñón, M. J.; Marroni, N. P. Role of quercetin in preventing thioacetamide-induced liver injury in rats. Toxicol. Pathol. 2011, 39, 949-957.

[25] Surapaneni, K. M.; Jainu, M. Pioglitazone, quercetin and hydroxy citric acid effect on hepatic biomarkers in non alcoholic steatohepatitis. Pharmacognosy Res. 2014, 6, 153-162.

[26] Surapaneni, K. M.; Priya, V. V.; Mallika, J. Pioglitazone, quercetin and hydroxy citric acid effect on cytochrome P450 2E1 (CYP2E1) enzyme levels in experimentally induced non alcoholic steatohepatitis (NASH). Eur. Rev. Med. Pharmacol. Sci. 2014, 18, 2736-2741.

[27] Li, X.; Wang, R.; Zhou, N.; Wang, X.; Liu, Q.; Bai, Y.; Bai, Y.; Liu, Z.; Yang, H.; Zou, J.; Wang, H.; Shi, T. Quercetin improves insulin resistance and hepatic lipid accumulation in a NAFLD cell model. Biomed. Rep. 2013, 1, 71-76.

[28] Vidyashankar, S.; Sandeep Varma, R.; Patki, P. S. Quercetin ameliorate insulin resistance and upregulates cellular antioxidants during oleic acid induced hepatic steatosis in HepG2 cells. Toxicol. In. Vitro. 2013, 27, 945-953.

[29] Panchal, S. K.; Poudyal, H.; Brown, L. Quercetin ameliorates cardiovascular, hepatic, and metabolic changes in diet-induced metabolic syndrome in rats. J. Nutr. 2012, 142, 1026-1032. 
[30] Jung, C. H.; Cho, I.; Ahn, J.; Jeon, T. I.; Ha, T. Y. Quercetin reduces high-fat diet-induced fat accumulation in the liver by regulating lipid metabolism genes. Phytother. Res. 2013, 27, 139-143.

[31] Zhang, M. H.; Liang, Z. Q.; Qin, Q.; Li, S. L.; Zhou, D. S.; Tang, L. Effects of quercetin on serum levels of resistin and IL-18 and on insulin resistance in nonalcoholic fatty liver disease rats. Zhonghua Gan Zang Bing Za Zhi 2013, 21, 66-70.

[32] Wang, W.; Wang, C.; Ding, X. Q.; Pan, Y.; Gu, T. T.; Wang, M. X.; Liu, Y. L.; Wang, F. M.; Wang, S. J.; Kong, L. D. Quercetin and allopurinol reduce liver thioredoxin-interacting protein to alleviate inflammation and lipid accumulation in diabetic rats. Br. J. Pharmacol. 2013, 169, 13521371.

[33] Zhang, D.; Xie, L.; Jia, G.; Cai, S.; Ji, B.; Liu, Y.; Wu, W.; Zhou, F.; Wang, A.; Chu, L.; Wei, Y.; Liu, J.; Gao, F. Comparative study on antioxidant capacity of flavonoids and their inhibitory effects on oleic acid-induced hepatic steatosis in vitro. Eur. J. Med. Chem. 2011, 46, 4548-4558.

[34] Zhou, D. S.; Liang, Z. Q.; Qin, Q.; Zhang, M. H.; Li, S. L. Therapeutic efficacy and mechanisms of quercetin in a rat model of nonalcoholic fatty liver disease. Zhonghua Gan Zang Bing Za Zhi 2013, 21, 134-137.

[35] Das, N.; Sikder, K.; Bhattacharjee, S.; Majumdar, S. B.; Ghosh, S.; Majumdar, S.; Dey, S. Quercetin alleviates inflammation after short-term treatment in high-fat-fed mice. Food Funct. 2013, 4, 889-898.

[36] Wang, X.; Zhang, D. M.; Gu, T. T.; Ding, X. Q.; Fan, C. Y.; Zhu, Q.; Shi, Y. W.; Hong, Y.; Kong, L. D. Morin reduces hepatic inflammation-associated lipid accumulation in high fructose-fed rats via inhibiting sphingosine kinase 1/sphingosine 1-phosphate signaling pathway. Biochem. Pharmacol. 2013, 86, 1791-1804.

[37] Panchal, S. K.; Poudyal, H.; Arumugam, T. V.; Brown, L. Rutin attenuates metabolic changes, nonalcoholic steatohepatitis, and cardiovascular remodeling in high-carbohydrate, high-fat diet-fed rats. J. Nutr. 2011, 141, 1062-1069.

[38] Zhang, Z. F.; Fan, S. H.; Zheng, Y. L.; Lu, J.; Wu, D. M.; Shan, Q.; Hu, B. Troxerutin improves hepatic lipid homeostasis by restoring $\mathrm{NAD}(+)$-depletion-mediated dysfunction of lipin 1 signaling in high-fat diet-treated mice. Biochem. Pharmacol. 2014, 91, 74-86.

[39] Li, X. Y.; Kong, L. X.; Li, J.; He, H. X.; Zhou, Y. D. Kaempferol suppresses lipid accumulation in macrophages through the downregulation of cluster of differentiation 36 and the upregulation of scavenger receptor class B type I and ATP-binding cassette transporters A1 and G1. Int. J. Mol. Med. 2013, 31, 331-338.

[40] Zhang, N.; Pei, F.; Wei, H.; Zhang, T.; Yang, C.; Ma, G.; Yang, C. Isorhamnetin protects rat ventricular myocytes from ischemia and reperfusion injury. Exp. Toxicol. Pathol. 2011, 63, 33-38.

[41] Kong, C. S.; Seo, Y. Antiadipogenic activity of isohamnetin 3-O-beta-D-glucopyranoside from Salicornia herbacea. Immunopharmacol. Immunotoxicol. 2012, 34, 907-911.

[42] Ramachandran, L.; Manu, K. A.; Shanmugam, M. K.; Li, F.; Siveen, K. S.; Vali, S.; Kapoor, S.; Abbasi, T.; Surana, R.; Smoot, D. T.; Ashktorab, H.; Tan, P.; Ahn, K. S.; Yap, C. W.; Kumar, A. P.; Sethi, G. Isorhamnetin inhibits proliferation and invasion and induces apoptosis through the 
modulation of peroxisome proliferator-activated receptor gamma activation pathway in gastric cancer. J. Biol. Chem. 2012, 287, 38028-38040.

[43] Chirumbolo, S. Anti-inflammatory action of isorhamnetin. Inflammation 2014, 37, 1200-1201.

[44] Dong, G. Z.; Lee, J. H.; Ki, S. H.; Yang, J. H.; Cho, I. J.; Kang, S. H.; Zhao, R. J.; Kim, S. C.; Kim, Y. W. AMPK activation by isorhamnetin protects hepatocytes against oxidative stress and mitochondrial dysfunction. Eur. J. Pharmacol. 2014, 740, 634-640.

[45] Choi, H. N.; Kang, M. J.; Lee, S. J.; Kim, J. I. Ameliorative effect of myricetin on insulin resistance in mice fed a high-fat, high-sucrose diet. Nutr. Res. Pract. 2014, 8, 544-549.

[46] Chang, C. J.; Tzeng, T. F.; Liou, S. S.; Chang, Y. S.; Liu, I. M. Myricetin increases hepatic peroxisome proliferator-activated receptor alpha protein expression and decreases plasma lipids and adiposity in rats. Evid Based. Complement. Alternat Med. 2012, 2012, 787152.

[47] Jung, C. H.; Jang, S. J.; Ahn, J.; Gwon, S. Y.; Jeon, T. I.; Kim, T. W.; Ha, T. Y. Alpinia officinarum inhibits adipocyte differentiation and high-fat diet-induced obesity in mice through regulation of adipogenesis and lipogenesis. J. Med. Food 2012, 15, 959-967.

[48] Jiang, Y.; Gong, F. L.; Zhao, G. B.; Li, J. Chrysin suppressed inflammatory responses and the inducible nitric oxide synthase pathway after spinal cord injury in rats. Int. J. Mol. Sci. 2014, 15, 12270-12279.

[49] Yang, B.; Huang, J.; Xiang, T.; Yin, X.; Luo, X.; Huang, J.; Luo, F.; Li, H.; Li, H.; Ren, G. Chrysin inhibits metastatic potential of human triple-negative breast cancer cells by modulating matrix metalloproteinase-10, epithelial to mesenchymal transition, and PI3K/Akt signaling pathway. J. Appl. Toxicol. 2014, 34, 105-112.

[50] Sirovina, D.; Orsolic, N.; Koncic, M. Z.; Kovacevic, G.; Benkovic, V.; Gregorovic, G. Quercetin vs chrysin: effect on liver histopathology in diabetic mice. Hum. Exp. Toxicol. 2013, 32, 10581066.

[51] Liang, Y. C.; Tsai, S. H.; Tsai, D. C.; Lin-Shiau, S. Y.; Lin, J. K. Suppression of inducible cyclooxygenase and nitric oxide synthase through activation of peroxisome proliferator-activated receptor-gamma by flavonoids in mouse macrophages. FEBS Lett. 2001, 496, 12-18.

[52] Feng, X.; Qin, H.; Shi, Q.; Zhang, Y.; Zhou, F.; Wu, H.; Ding, S.; Niu, Z.; Lu, Y.; Shen, P. Chrysin attenuates inflammation by regulating M1/M2 status via activating PPARgamma. Biochem. Pharmacol. 2014, 89, 503-514.

[53] Wang, J.; Liu, Y. T.; Xiao, L.; Zhu, L.; Wang, Q.; Yan, T. Anti-inflammatory effects of apigenin in lipopolysaccharide-induced inflammatory in acute lung injury by suppressing COX-2 and NFkB pathway. Inflammation 2014.

[54] Zhang, X.; Wang, G.; Gurley, E. C.; Zhou, H. Flavonoid apigenin inhibits lipopolysaccharideinduced inflammatory response through multiple mechanisms in macrophages. PLoS One 2014, 9, e107072.

[55] Tong, X.; Pelling, J. C. Targeting the PI3K/Akt/mTOR axis by apigenin for cancer prevention. Anticancer Agents Med. Chem. 2013, 13, 971-978.

[56] Bao, Y. Y.; Zhou, S. H.; Fan, J.; Wang, Q. Y. Anticancer mechanism of apigenin and the implications of GLUT-1 expression in head and neck cancers. Future Oncol. 2013, 9, 1353-1364. 
[57] Lefort, E. C.; Blay, J. Apigenin and its impact on gastrointestinal cancers. Mol. Nutr. Food Res. 2013, 57, 126-144.

[58] Bumke-Vogt, C.; Osterhoff, M. A.; Borchert, A.; Guzman-Perez, V.; Sarem, Z.; Birkenfeld, A. L.; Bahr, V.; Pfeiffer, A. F. The flavones apigenin and luteolin induce FOXO1 translocation but inhibit gluconeogenic and lipogenic gene expression in human cells. PLoS One 2014, 9, e104321.

[59] López-Lázaro, M. Distribution and biological activities of the flavonoid luteolin. Mini Rev. Med. Chem. 2009, 9, 31-59.

[60] Chen, D.; Bi, A.; Dong, X.; Jiang, Y.; Rui, B.; Liu, J.; Yin, Z.; Luo, L. Luteolin exhibits antiinflammatory effects by blocking the activity of heat shock protein 90 in macrophages. Biochem. Biophys. Res. Commun. 2014, 443, 326-332.

[61] Kim, Y. S.; Kim, S. H.; Shin, J.; Harikishore, A.; Lim, J. K.; Jung, Y.; Lyu, H. N.; Baek, N. I.; Choi, K. Y.; Yoon, H. S.; Kim, K. T. Luteolin suppresses cancer cell proliferation by targeting vaccinia-related kinase 1. PLoS One 2014, 9, e109655.

[62] Osman, N. H.; Said, U. Z.; El-Waseef, A. M.; Ahmed, E. S. Luteolin supplementation adjacent to aspirin treatment reduced dimethylhydrazine-induced experimental colon carcinogenesis in rats. Tumour Biol. 2014.

[63] Liu, J. F.; Ma, Y.; Wang, Y.; Du, Z. Y.; Shen, J. K.; Peng, H. L. Reduction of lipid accumulation in HepG2 cells by luteolin is associated with activation of AMPK and mitigation of oxidative stress. Phytother. Res. 2011, 25, 588-596.

[64] Mulvihill, E. E.; Allister, E. M.; Sutherland, B. G.; Telford, D. E.; Sawyez, C. G.; Edwards, J. Y.; Markle, J. M.; Hegele, R. A.; Huff, M. W. Naringenin prevents dyslipidemia, apolipoprotein B overproduction, and hyperinsulinemia in LDL receptor-null mice with diet-induced insulin resistance. Diabetes 2009, 58, 2198-2210.

[65] Esmaeili, A. H.; Hajizadeh Moghaddam, A.; Chaichi, M. J. Identification, determination, and study of antioxidative activities of hesperetin and gallic acid in hydro-alcoholic extract from flowers of Eriobotrya japonica (Lindl.). Avicenna J. Phytomed 2014, 4, 260-266.

[66] Fordham, J. B.; Naqvi, A. R.; Nares, S. Leukocyte production of inflammatory mediators is inhibited by the antioxidants phloretin, silymarin, hesperetin, and resveratrol. Mediators Inflamm. 2014, 2014, 938712.

[67] Palit, S.; Kar, S.; Sharma, G.; Das, P. K. Hesperetin induces apoptosis in breast carcinoma by triggering accumulation of ROS and activation of ASK1/JNK pathway. J. Cell. Physiol. 2014.

[68] Lee, N. K.; Choi, S. H.; Park, S. H.; Park, E. K.; Kim, D. H. Antiallergic activity of hesperidin is activated by intestinal microflora. Pharmacology 2004, 71, 174-180.

[69] Choi, E. J.; Ahn, W. S. Neuroprotective effects of chronic hesperetin administration in mice. Arch. Pharm. Res. 2008, 31, 1457-1462.

[70] Casaschi, A.; Rubio, B. K.; Maiyoh, G. K.; Theriault, A. G. Inhibitory activity of diacylglycerol acyltransferase (DGAT) and microsomal triglyceride transfer protein (MTP) by the flavonoid, taxifolin, in HepG2 cells: potential role in the regulation of apolipoprotein B secretion. Atherosclerosis 2004, 176, 247-253. 
[71] Brusselmans, K.; Vrolix, R.; Verhoeven, G.; Swinnen, J. V. Induction of cancer cell apoptosis by flavonoids is associated with their ability to inhibit fatty acid synthase activity. J. Biol. Chem. 2005, 280, 5636-5645.

[72] Kochi, T.; Shimizu, M.; Terakura, D.; Baba, A.; Ohno, T.; Kubota, M.; Shirakami, Y.; Tsurumi, H.; Tanaka, T.; Moriwaki, H. Non-alcoholic steatohepatitis and preneoplastic lesions develop in the liver of obese and hypertensive rats: suppressing effects of EGCG on the development of liver lesions. Cancer Lett. 2014, 342, 60-69.

[73] Xiao, J.; Ho, C. T.; Liong, E. C.; Nanji, A. A.; Leung, T. M.; Lau, T. Y.; Fung, M. L.; Tipoe, G. L. Epigallocatechin gallate attenuates fibrosis, oxidative stress, and inflammation in non-alcoholic fatty liver disease rat model through TGF/SMAD, PI3 K/Akt/FoxO1, and NF-kappa B pathways. Eur. J. Nutr. 2014, 53, 187-199.

[74] Shimada, T.; Tokuhara, D.; Tsubata, M.; Kamiya, T.; Kamiya-Sameshima, M.; Nagamine, R.; Takagaki, K.; Sai, Y.; Miyamoto, K.; Aburada, M. Flavangenol (pine bark extract) and its major component procyanidin B1 enhance fatty acid oxidation in fat-loaded models. Eur. J. Pharmacol. 2012, 677, 147-153.

[75] Kim, M. H.; Kang, K. S. Isoflavones as a smart curer for non-alcoholic fatty liver disease and pathological adiposity via ChREBP and Wnt signaling. Prev. Med. 2012, 54 Suppl, S57-63.

[76] Westmark, C. J. A hypothesis regarding the molecular mechanism underlying dietary soy-induced effects on seizure propensity. Front. Neurol. 2014, 5, 169.

[77] Kim, M. H.; Park, J. S.; Jung, J. W.; Byun, K. W.; Kang, K. S.; Lee, Y. S. Daidzein supplementation prevents non-alcoholic fatty liver disease through alternation of hepatic gene expression profiles and adipocyte metabolism. Int. J. Obes. (Lond) 2011, 35, 1019-1030.

[78] Zheng, P. Y.; Ma, Z. S.; Hua, Y. Q.; Liu, T.; Xing, L. J.; Ji, G. Effects of puerarin on expressions of leptin receptor mRNA, phosphorylated Janus kinase 2/phosphorylated signal transducers and activators of transcription 3 proteins in the liver of rats with non-alcoholic fatty liver. Zhong Xi Yi Jie Не Хие Вао 2008, 6, 921-927.

[79] Zheng, P.; Ji, G.; Ma, Z.; Liu, T.; Xin, L.; Wu, H.; Liang, X.; Liu, J. Therapeutic effect of puerarin on non-alcoholic rat fatty liver by improving leptin signal transduction through JAK2/STAT3 pathways. Am. J. Chin. Med. 2009, 37, 69-83.

[80] Wang, Y.; Li, J.; Zhuge, L.; Su, D.; Yang, M.; Tao, S.; Li, J. Comparison between the efficacies of curcumin and puerarin in C57BL/6 mice with steatohepatitis induced by a methionine- and choline-deficient diet. Exp. Ther. Med. 2014, 7, 663-668.

[81] Mohamed Salih, S.; Nallasamy, P.; Muniyandi, P.; Periyasami, V.; Carani Venkatraman, A. Genistein improves liver function and attenuates non-alcoholic fatty liver disease in a rat model of insulin resistance. J. Diabetes 2009, 1, 278-287.

[82] Kim, M. H.; Kang, K. S.; Lee, Y. S. The inhibitory effect of genistein on hepatic steatosis is linked to visceral adipocyte metabolism in mice with diet-induced non-alcoholic fatty liver disease. $\mathrm{Br}$. $\mathrm{J}$. Nutr. 2010, 104, 1333-1342. 
[83] Shih, P. H.; Hwang, S. L.; Yeh, C. T.; Yen, G. C. Synergistic effect of cyanidin and PPAR agonist against nonalcoholic steatohepatitis-mediated oxidative stress-induced cytotoxicity through MAPK and Nrf2 transduction pathways. J. Agric. Food Chem. 2012, 60, 2924-2933.

[84] Wei, X.; Wang, D.; Yang, Y.; Xia, M.; Li, D.; Li, G.; Zhu, Y.; Xiao, Y.; Ling, W. Cyanidin-3-Obeta-glucoside improves obesity and triglyceride metabolism in KK-Ay mice by regulating lipoprotein lipase activity. J. Sci. Food Agric. 2011, 91, 1006-1013.

[85] Guo, H.; Li, D.; Ling, W.; Feng, X.; Xia, M. Anthocyanin inhibits high glucose-induced hepatic mtGPAT1 activation and prevents fatty acid synthesis through PKCzeta. J. Lipid Res. 2011, 52, 908-922.

[86] Jiang, X.; Tang, X.; Zhang, P.; Liu, G.; Guo, H. Cyanidin-3-O-beta-glucoside protects primary mouse hepatocytes against high glucose-induced apoptosis by modulating mitochondrial dysfunction and the PI3K/Akt pathway. Biochem. Pharmacol. 2014, 90, 135-144.

[87] Wu, T.; Qi, X.; Liu, Y.; Guo, J.; Zhu, R.; Chen, W.; Zheng, X.; Yu, T. Dietary supplementation with purified mulberry (Morus australis Poir) anthocyanins suppresses body weight gain in highfat diet fed C57BL/6 mice. Food Chem. 2013, 141, 482-487.

[88] Valenti, L.; Riso, P.; Mazzocchi, A.; Porrini, M.; Fargion, S.; Agostoni, C. Dietary anthocyanins as nutritional therapy for nonalcoholic fatty liver disease. Oxid Med. Cell. Longev 2013, 2013, 145421.

[89] Mao, Z. M.; Song, H. Y.; Yang, L. L.; Liu, T.; Li, D. F.; Zheng, P. Y.; Liu, P.; Ji, G. Effects of the mixture of Swertia pseudochinensis Hara and Silybum marianum Gaertn extracts on $\mathrm{CCl}(4)$ induced liver injury in rats with non-alcoholic fatty liver disease. Zhong Xi Yi Jie He Xue Bao 2012, 10, 193-199.

[90] Zhang, S.; Zheng, L.; Dong, D.; Xu, L.; Yin, L.; Qi, Y.; Han, X.; Lin, Y.; Liu, K.; Peng, J. Effects of flavonoids from Rosa laevigata Michx fruit against high-fat diet-induced non-alcoholic fatty liver disease in rats. Food Chem. 2013, 141, 2108-2116.

[91] Salamone, F.; Galvano, F.; Cappello, F.; Mangiameli, A.; Barbagallo, I.; Li Volti, G. Silibinin modulates lipid homeostasis and inhibits nuclear factor kappa B activation in experimental nonalcoholic steatohepatitis. Transl. Res. 2012, 159, 477-486.

[92] Salamone, F.; Galvano, F.; Marino Gammazza, A.; Paternostro, C.; Tibullo, D.; Bucchieri, F.; Mangiameli, A.; Parola, M.; Bugianesi, E.; Li Volti, G. Silibinin improves hepatic and myocardial injury in mice with nonalcoholic steatohepatitis. Dig. Liver Dis. 2012, 44, 334-342.

[93] Yao, J.; Zhi, M.; Minhu, C. Effect of silybin on high-fat-induced fatty liver in rats. Braz. J. Med. Biol. Res. 2011, 44, 652-659.

[94] Li, Z. Z.; Berk, M.; McIntyre, T. M.; Feldstein, A. E. Hepatic lipid partitioning and liver damage in nonalcoholic fatty liver disease: role of stearoyl-CoA desaturase. J. Biol. Chem. 2009, 284, 5637-5644.

[95] Guzmán, C.; Benet, M.; Pisonero-Vaquero, S.; Moya, M.; García-Mediavilla, M. V.; MartínezChantar, M. L.; González-Gallego, J.; Castell, J. V.; Sánchez-Campos, S.; Jover, R. The human liver fatty acid binding protein (FABP1) gene is activated by FOXA1 and PPARalpha; and 
repressed by $\mathrm{C} / \mathrm{EBPalpha:} \mathrm{Implications} \mathrm{in} \mathrm{FABP1} \mathrm{down-regulation} \mathrm{in} \mathrm{nonalcoholic} \mathrm{fatty} \mathrm{liver}$ disease. Biochim. Biophys. Acta 2013, 1831, 803-818.

[96] Aghazadeh, S.; Amini, R.; Yazdanparast, R.; Ghaffari, S. H. Anti-apoptotic and anti-inflammatory effects of Silybum marianum in treatment of experimental steatohepatitis. Exp. Toxicol. Pathol. 2011, 63, 569-574.

[97] Kim, M.; Yang, S. G.; Kim, J. M.; Lee, J. W.; Kim, Y. S.; Lee, J. I. Silymarin suppresses hepatic stellate cell activation in a dietary rat model of non-alcoholic steatohepatitis: analysis of isolated hepatic stellate cells. Int. J. Mol. Med. 2012, 30, 473-479.

[98] Buturova, L. I.; Tsybizova, T. A.; Kalinin, A. V. Use of Legalon in non-alcoholic fatty liver disease. Eksp. Klin. Gastroenterol. 2010, (5), 69-75.

[99] Cacciapuoti, F.; Scognamiglio, A.; Palumbo, R.; Forte, R.; Cacciapuoti, F. Silymarin in non alcoholic fatty liver disease. World J. Hepatol. 2013, 5, 109-113.

[100] Schrieber, S. J.; Hawke, R. L.; Wen, Z.; Smith, P. C.; Reddy, K. R.; Wahed, A. S.; Belle, S. H.; Afdhal, N. H.; Navarro, V. J.; Meyers, C. M.; Doo, E.; Fried, M. W. Differences in the disposition of silymarin between patients with nonalcoholic fatty liver disease and chronic hepatitis C. Drug Metab. Dispos. 2011, 39, 2182-2190.

[101] Kidd, P. M. Bioavailability and activity of phytosome complexes from botanical polyphenols: the silymarin, curcumin, green tea, and grape seed extracts. Altern. Med. Rev. 2009, 14, 226-246.

[102] Loguercio, C.; Andreone, P.; Brisc, C.; Brisc, M. C.; Bugianesi, E.; Chiaramonte, M.; Cursaro, C.; Danila, M.; de Sio, I.; Floreani, A.; Freni, M. A.; Grieco, A.; Groppo, M.; Lazzari, R.; Lobello, S.; Lorefice, E.; Margotti, M.; Miele, L.; Milani, S.; Okolicsanyi, L.; Palasciano, G.; Portincasa, P.; Saltarelli, P.; Smedile, A.; Somalvico, F.; Spadaro, A.; Sporea, I.; Sorrentino, P.; Vecchione, R.; Tuccillo, C.; Del Vecchio Blanco, C.; Federico, A. Silybin combined with phosphatidylcholine and vitamin $\mathrm{E}$ in patients with nonalcoholic fatty liver disease: a randomized controlled trial. Free Radic. Biol. Med. 2012, 52, 1658-1665.

[103] Iakimchuk, G. N.; Gendrikson, L. N. Study of clinical efficiency of essential phospholipids and silymarin combination in nonalcoholic and alcoholic steatohepatitis. Eksp. Klin. Gastroenterol. 2011, (7), 64-69.

[104] Federico, A.; Trappoliere, M.; Tuccillo, C.; de Sio, I.; Di Leva, A.; Del Vecchio Blanco, C.; Loguercio, C. A new silybin-vitamin E-phospholipid complex improves insulin resistance and liver damage in patients with non-alcoholic fatty liver disease: preliminary observations. Gut 2006, 55, 901-902.

[105] Trappoliere, M.; Federico, A.; Tuccillo, C.; de Sio, I.; Di Leva, A.; Niosi, M.; D'Auria, M.; Loguercio, C.; Real Sud Group 2 Effects of a new pharmacological complex (silybin + vitamin-E + phospholipids) on some markers of the metabolic syndrome and of liver fibrosis in patients with hepatic steatosis. Preliminary study. Minerva Gastroenterol. Dietol. 2005, 51, 193-199.

[106] Zeng, C. H.; Zeng, P.; Deng, Y. H.; Shen, N.; Peng, M. L.; Liu, Q.; Ren, H. The effects of curcumin derivative on experimental steatohepatitis. Zhonghua Gan Zang Bing Za Zhi 2011, 19, 454-459. 
[107] Kuo, J. J.; Chang, H. H.; Tsai, T. H.; Lee, T. Y. Positive effect of curcumin on inflammation and mitochondrial dysfunction in obese mice with liver steatosis. Int. J. Mol. Med. 2012, 30, 673-679.

[108] Kang, O. H.; Kim, S. B.; Seo, Y. S.; Joung, D. K.; Mun, S. H.; Choi, J. G.; Lee, Y. M.; Kang, D. G.; Lee, H. S.; Kwon, D. Y. Curcumin decreases oleic acid-induced lipid accumulation via AMPK phosphorylation in hepatocarcinoma cells. Eur. Rev. Med. Pharmacol. Sci. 2013, 17, 2578-2586.

[109] Kuo, J. J.; Chang, H. H.; Tsai, T. H.; Lee, T. Y. Curcumin ameliorates mitochondrial dysfunction associated with inhibition of gluconeogenesis in free fatty acid-mediated hepatic lipoapoptosis. Int. J. Mol. Med. 2012, 30, 643-649.

[110] Messner, D. J.; Rhieu, B. H.; Kowdley, K. V. Iron overload causes oxidative stress and impaired insulin signaling in AML-12 hepatocytes. Dig. Dis. Sci. 2013, 58, 1899-1908.

[111] Zhu, W.; Chen, S.; Li, Z.; Zhao, X.; Li, W.; Sun, Y.; Zhang, Z.; Ling, W.; Feng, X. Effects and mechanisms of resveratrol on the amelioration of oxidative stress and hepatic steatosis in KKAy mice. Nutr. Metab. (Lond) 2014, 11, 35-7075-11-35. eCollection 2014.

[112] Aguirre, L.; Portillo, M. P.; Hijona, E.; Bujanda, L. Effects of resveratrol and other polyphenols in hepatic steatosis. World J. Gastroenterol. 2014, 20, 7366-7380.

[113] Aoun, M.; Michel, F.; Fouret, G.; Casas, F.; Jullien, M.; Wrutniak-Cabello, C.; Ramos, J.; Cristol, J. P.; Coudray, C.; Carbonneau, M. A.; Feillet-Coudray, C. A polyphenol extract modifies quantity but not quality of liver fatty acid content in high-fat-high-sucrose diet-fed rats: possible implication of the sirtuin pathway. Br. J. Nutr. 2010, 104, 1760-1770.

[114] Panchal, S. K.; Brown, L. Cardioprotective and hepatoprotective effects of ellagitannins from European oak bark (Quercus petraea L.) extract in rats. Eur. J. Nutr. 2013, 52, 397-408.

[115] Luo, X. Y.; Takahara, T.; Hou, J.; Kawai, K.; Sugiyama, T.; Tsukada, K.; Takemoto, M.; Takeuchi, M.; Zhong, L.; Li, X. K. Theaflavin attenuates ischemia-reperfusion injury in a mouse fatty liver model. Biochem. Biophys. Res. Commun. 2012, 417, 287-293.

[116] Chung, M. Y.; Park, H. J.; Manautou, J. E.; Koo, S. I.; Bruno, R. S. Green tea extract protects against nonalcoholic steatohepatitis in ob/ob mice by decreasing oxidative and nitrative stress responses induced by proinflammatory enzymes. J. Nutr. Biochem. 2012, 23, 361-367.

[117] Park, H. J.; Lee, J. Y.; Chung, M. Y.; Park, Y. K.; Bower, A. M.; Koo, S. I.; Giardina, C.; Bruno, R. S. Green tea extract suppresses NFkappaB activation and inflammatory responses in dietinduced obese rats with nonalcoholic steatohepatitis. J. Nutr. 2012, 142, 57-63.

[118] Sakata, R.; Nakamura, T.; Torimura, T.; Ueno, T.; Sata, M. Green tea with high-density catechins improves liver function and fat infiltration in non-alcoholic fatty liver disease (NAFLD) patients: a double-blind placebo-controlled study. Int. J. Mol. Med. 2013, 32, 989-994.

[119] Masterjohn, C.; Bruno, R. S. Therapeutic potential of green tea in nonalcoholic fatty liver disease. Nutr. Rev. 2012, 70, 41-56.

[120] Jung, J. H.; Kim, H. S. The inhibitory effect of black soybean on hepatic cholesterol accumulation in high cholesterol and high fat diet-induced non-alcoholic fatty liver disease. Food Chem. Toxicol. 2013, 60, 404-412. 
[121] Badger, T. M.; Ronis, M. J.; Wolff, G.; Stanley, S.; Ferguson, M.; Shankar, K.; Simpson, P.; Jo, C. H. Soy protein isolate reduces hepatosteatosis in yellow Avy/a mice without altering coat color phenotype. Exp. Biol. Med. (Maywood) 2008, 233, 1242-1254.

[122] Leng, L.; Jiang, Z. Q.; Ji, G. Y. Effects of soybean isoflavone on liver lipid metabolism in nonalcoholic fatty liver rats. Zhonghua Yu Fang Yi Xue Za Zhi 2011, 45, 335-339.

[123] Davaatseren, M.; Hur, H. J.; Yang, H. J.; Hwang, J. T.; Park, J. H.; Kim, H. J.; Kim, M. J.; Kwon, D. Y.; Sung, M. J. Taraxacum official (dandelion) leaf extract alleviates high-fat diet-induced nonalcoholic fatty liver. Food Chem. Toxicol. 2013, 58, 30-36.

[124] Davaatseren, M.; Hur, H. J.; Yang, H. J.; Hwang, J. T.; Park, J. H.; Kim, H. J.; Kim, M. S.; Kim, M. J.; Kwon, D. Y.; Sung, M. J. Dandelion leaf extract protects against liver injury induced by methionine- and choline-deficient diet in mice. J. Med. Food 2013, 16, 26-33.

[125] Vareda, P. M.; Saldanha, L. L.; Camaforte, N. A.; Violato, N. M.; Dokkedal, A. L.; Bosqueiro, J. R. Myrcia bella leaf extract presents hypoglycemic activity via PI3k/Akt insulin signaling pathway. Evid Based. Complement. Alternat Med. 2014, 2014, 543606.

[126] Shen, B.; Yu, J.; Wang, S.; Chu, E. S.; Wong, V. W.; Zhou, X.; Lin, G.; Sung, J. J.; Chan, H. L. Phyllanthus urinaria ameliorates the severity of nutritional steatohepatitis both in vitro and in vivo. Hepatology 2008, 47, 473-483.

[127] Yang, J.; Lv, F.; Chen, X. Q.; Cui, W. X.; Chen, L. H.; Wen, X. D.; Wang, Q. Pharmacokinetic study of major bioactive components in rats after oral administration of extract of Ilex hainanensis by high-performance liquid chromatography/electrospray ionization mass spectrometry. J. Pharm. Biomed. Anal. 2013, 77, 21-28.

[128] Cui, W. X.; Yang, J.; Chen, X. Q.; Mao, Q.; Wei, X. L.; Wen, X. D.; Wang, Q. Triterpenoid-rich fraction from Ilex hainanensis Merr. attenuates non-alcoholic fatty liver disease induced by high fat diet in rats. Am. J. Chin. Med. 2013, 41, 487-502.

[129] Li, J. J.; Yang, J.; Cui, W. X.; Chen, X. Q.; Chen, G. L.; Wen, X. D.; Wang, Q. Analysis of therapeutic effect of Ilex hainanensis Merr. extract on nonalcoholic fatty liver disease through urine metabolite profiling by ultraperformance liquid chromatography/quadrupole time of flight mass spectrometry. Evid Based. Complement. Alternat Med. 2013, 2013, 451975.

[130] Wang, S. D.; Xie, Z. Q.; Chen, J.; Wang, K.; Wei, T.; Zhao, A. H.; Zhang, Q. H. Inhibitory effect of Ginkgo biloba extract on fatty liver: regulation of carnitine palmitoyltransferase 1a and fatty acid metabolism. J. Dig. Dis. 2012, 13, 525-535.

[131] Wei, T.; Xiong, F. F.; Wang, S. D.; Wang, K.; Zhang, Y. Y.; Zhang, Q. H. Flavonoid ingredients of. J. Biomed. Sci. 2014, 21, 87.

[132] Zhang, L.; Xu, J.; Song, H.; Yao, Z.; Ji, G. Extracts from Salvia-Nelumbinis naturalis alleviate hepatosteatosis via improving hepatic insulin sensitivity. J. Transl. Med. 2014, 12, 236-014-02368.

[133] Yin, J.; Luo, Y.; Deng, H.; Qin, S.; Tang, W.; Zeng, L.; Zhou, B. Hugan Qingzhi medication ameliorates hepatic steatosis by activating AMPK and PPARalpha pathways in L02 cells and HepG2 cells. J. Ethnopharmacol. 2014, 154, 229-239. 
[134] Chen, Z. Y.; Li, J. S.; Jiang, J. P.; Yan, M. X.; He, B. H. Effect of pure total flavonoids from citrus on hepatic SIRT1/PGC-1alpha pathway in mice with NASH. Zhongguo Zhong Yao Za Zhi 2014, 39, 100-105.

[135] Wei, Y. Y.; Yan, D.; Japar, A.; Qu, S. S.; Haji, A. A.; Parhat, K. Effects of pomegranate flowers polyphenols on liver PON expression of diabetes combining non-alcoholic fat liver diseases rats. Yao Xиe Хие Bao 2013, 48, 71-76.

[136] Liu, Y.; Wang, D.; Zhang, D.; Lv, Y.; Wei, Y.; Wu, W.; Zhou, F.; Tang, M.; Mao, T.; Li, M.; Ji, B. Inhibitory effect of blueberry polyphenolic compounds on oleic acid-induced hepatic steatosis in vitro. J. Agric. Food Chem. 2011, 59, 12254-12263.

[137] Wu, T.; Tang, Q.; Gao, Z.; Yu, Z.; Song, H.; Zheng, X.; Chen, W. Blueberry and mulberry juice prevent obesity development in C57BL/6 mice. PLoS One 2013, 8, e77585.

[138] Guo, H.; Zhong, R.; Liu, Y.; Jiang, X.; Tang, X.; Li, Z.; Xia, M.; Ling, W. Effects of bayberry juice on inflammatory and apoptotic markers in young adults with features of non-alcoholic fatty liver disease. Nutrition 2014, 30, 198-203.

[139] Tsuruta, Y.; Nagao, K.; Kai, S.; Tsuge, K.; Yoshimura, T.; Koganemaru, K.; Yanagita, T. Polyphenolic extract of lotus root (edible rhizome of Nelumbo nucifera) alleviates hepatic steatosis in obese diabetic db/db mice. Lipids Health. Dis. 2011, 10, 202-511X-10-202. 